\title{
Hypoxia induces ZEB2 in podocytes: Implications in the pathogenesis of proteinuria
}

\author{
Krishnamurthy Nakuluri $^{1}$ | Dhanunjay Mukhi ${ }^{1}$ | Rajkishor Nishad $^{1}$ | Moin A. Saleem ${ }^{2}$ | \\ Sathish Kumar Mungamuri $^{3}$ | Ram K. Menon ${ }^{4,5}$ | Anil Kumar Pasupulati ${ }^{1}$ (i)
}

${ }^{1}$ Department of Biochemistry, School of Life Sciences, University of Hyderabad,

Hyderabad, India

${ }^{2}$ Academic Renal Unit, University of Bristol, Bristol, UK

${ }^{3}$ Institute of Basic Sciences and Translational Research, Asian Health Care Foundation,

Asian Institute of Gastroenterology,

Hyderabad, India

${ }^{4}$ Department of Pediatrics, University of Michigan, Ann Arbor, Michigan

${ }^{5}$ Department of Molecular and Integrative Physiology, University of Michigan, Ann Arbor, Michigan

\section{Correspondence}

Anil Kumar Pasupulati, Department of Biochemistry, School of Life Sciences, University of Hyderabad, Gachibowli, Hyderabad 500046, India.

Email: pasupulati.anilkumar@gmail.com Sathish Kumar Mungamuri, Institute of Basic Sciences and Translational Research, Asian Health Care Foundation, Asian Institute of Gastroenterology, Somajiguda, Hyderabad 500082, India.

Email: mungamurisk@gmail.com

Funding information

Defence Research and Development

Organisation, India, Grant/Award Numbers: LSRB-296/PEE\&BS/2016, EMR/2015/002076; Life Science Research Board (LSRB) and Science and Engineering Research Board (SERB)

\begin{abstract}
The glomerular filtration barrier (GFB) plays a critical role in ensuing protein free urine. The integrity of the GFB is compromised during hypoxia that prevails during extreme physiological conditions. However, the mechanism by which glomerular permselectivity is compromised during hypoxia remains enigmatic. Rats exposed to hypoxia showed a decreased glomerular filtration rate, podocyte foot-processes effacement, and proteinuria. Accumulation of hypoxia-inducible factor-1 $\alpha$ (HIF1 $\alpha)$ in podocytes resulted in elevated expression of zinc finger E-box binding homeobox 2 (ZEB2) and decreased expression of E- and P-cadherin. We also demonstrated that HIF1 $\alpha$ binds to hypoxia response element localized in the ZEB2 promoter. Furthermore, HIF1 $\alpha$ also induced the expression of ZEB2-natural antisense transcript, which is known to increase the efficiency of ZEB2 translation. Ectopic expression of ZEB2 induced loss of E- and P-cadherin and is associated with enhanced motility of podocytes during hypoxic conditions. ZEB2 knockdown abrogated hypoxia-induced decrease in podocyte permselectivity. This study suggests that hypoxia leads to activation of HIF1 $\alpha$-ZEB2 axis, resulting in podocyte injury and poor renal outcome.
\end{abstract}

KEYWORDS

HIF1 $\alpha$, hypoxia, podocyte, proteinuria, ZEB2.

\section{1 | INTRODUCTION}

Oxygen is a vital element and supports most of the metabolic events in higher organisms. The continuous supply of adequate levels of oxygen is crucial for normal functioning of the human body. However, human physiology is challenged with extreme environmental conditions resulting in hypoxia, a state of deficiency of oxygen in the blood and tissues. Hypoxia affects the homeostasis and functioning of various organs including kidneys. Kidneys possess lowresistance microvasculature that is exposed to both high volume and continuous perfusion. The kidneys have a high oxygen demand, so as to facilitate energy dependent basic renal functions such as active salt absorption (Hansell, Welch, Blantz, \& Palm, 2013). The constraints of low oxygen supply, dictated by both renal architecture 
and high oxygen demand, coalesce to make the kidney vulnerable to physiologic, environmental, and pathological stresses that cause hypoxia. The limitation in renal tissue oxygen supply during chronic hypoxia exposes the kidneys to hypoxia-induced adaptations and has long been recognized as an important factor in the pathogenesis of acute renal injury (Eckardt et al., 2005; Goldfarb-Rumyantzev \& Alper, 2014; Kushida et al., 2016; Munshi, Hsu, \& Himmelfarb, 2011; S. Tanaka, Tanaka, \& Nangaku, 2016).

The vertebrate kidneys play an essential role in the maintenance of homeostasis via its role in the filtration of plasma and the regulation of water, electrolyte, and acid-base balance. The function of the kidneys is to ensure almost protein free ultrafiltrated urine. The presence of proteins in the urine (proteinuria) is an indication of renal injury, particularly aberrations in the glomerular filtration barrier (GFB). The crucial components of GFB include the glomerular capillary endothelium, basement membrane, and glomerular podocytes (Anil Kumar, Welsh, Saleem, \& Menon, 2014). Albuminuria is an index of adverse renal outcome that can be assessed by measuring albumin levels in urine. Microalbuminuria describes levels of urine albumin ranging from 30 to $300 \mathrm{mg} / 24 \mathrm{hr}$, and macroalbuminuria describes a urinary albumin excretion of $\geq 300 \mathrm{mg} / 24 \mathrm{hr}$. The condition of macroalbuminuria often progresses to overt proteinuria and even to end-stage renal disease (ESRD) and is driven by an array of interlinked risk factors such as anemia, atherosclerosis, hyperglycemia, hypertension, repeated episodes of acute kidney injury, smoking, stroke, and sleep apnea, so forth. It has been well established that all these factors subject the kidneys to hypoxia (Adeseun \& Rosas, 2010; Fu, Colgan, \& Shelley, 2016; Tao et al., 2016). It was proposed that chronic hypoxia is the common pathway that leads to the ESRD (Fine, Bandyopadhay, \& Norman, 2000) and progression of chronic renal disease (Eckardt et al., 2005; Fine et al., 2000; Fu et al., 2016; Munshi et al., 2011; Palm \& Nordquist, 2011; T. Tanaka, Miyata, Inagi, Fujita, \& Nangaku, 2004).

Population-based studies demonstrated that approximately $10-20 \%$ of adults have proteinuria in their lifetime (Chadban et al., 2003; Coresh, Astor, Greene, Eknoyan, \& Levey, 2003). The incidence of ESRD has already reached epidemic proportions in many countries. Furthermore, the rates of ESRD among the elderly are disproportionally high. Considering the magnitude of the population with proteinuria and association of proteinuria with the clinical conditions wherein hypoxia prevails, it is important to understand the underlying mechanism of renal dysfunction to develop preventive and therapeutic strategies. Our understanding of the physiological and cellular events that transduce hypoxia-mediated events to adverse glomerular function is very limiting. In the current study, we investigated the mechanism of renal dysfunction following chronic hypoxia in rats and found that hypoxia results in elevated expression of hypoxia-inducible factor- $1 \alpha$ (HIF $1 \alpha$ ) in the glomerular region. Elevated HIF1 $\alpha$ in turn induced zinc finger E-box binding homeobox 2 (ZEB2) expression, both by directly interacting with the ZEB2 promoter and by increasing expression of a ZEB2- natural antisense transcript (ZEB2-NAT). Ectopic expression of ZEB2 induced loss of $\mathrm{E}$ - and $\mathrm{P}$-cadherin and was associated with enhanced migration of cells. Finally, hypoxia ablated the podocyte permselectivity, whereas ZEB2 knockdown abrogated this effect. Elevated expression of ZEB2 is implicated in podocyte epithelial-mesenchymal transition (EMT) and foot-process effacement during acute hypoxia. We conclude that hyperactivity of the HIF1 $\alpha-Z E B 2$ axis is one of the mechanisms by which acute systemic hypoxia provokes poor renal outcome.

\section{2 | MATERIALS AND METHODS}

\subsection{Reagents and plasmids}

Antibodies for P-cadherin (\#sc-1501), WT1 (\#sc-192), nephrin (\#sc-376522), ZO-1 (\#sc-33725) were obtained from Santa Cruz Biotechnology (Dallas, TX); ZEB2 (\#PA5-20980) was purchased from Thermo Fisher Scientific (Waltham, MA). Actin (\#4970), CD2AP (\#2135), HIF1 $\alpha$ (\#14179, \#79233), N-cadherin (\#4061), MMP2 (\#4022), E-cadherin (\#3195), MMP9 (\#3852), MMP2 (\#4022), VEGF (\#9943), tissue transglutaminase (TG2; \#73612), Vimentin (\#5741), $\beta$-catenin (\#8480), Histone3 (\#4499), FSP1 (\#13018) were procured from Cell Signaling Technologies (Danvers, MA). Podocin antibody is a gift from Rakesh Verma (University of Michigan, Ann Arbor, MI). Secondary antibodies were purchased from The Jackson Laboratory (Bar Harbor, ME). All primers used in this study procured from Integrated DNA Technologies (Coralville, IA). RNA isolation kit was purchased from Qiagen (Hilden, Germany). The high capacity cDNA reverse transcription kit, SYBR Green Master Mix, and enhanced chemiluminescence $(E C L)$ western blot analysis reagents were obtained from Bio-Rad Laboratories (Hercules, CA). Fetal bovine serum, RPMI1640, and TRIzol reagent were procured from Life Technologies (Carlsbad, CA). Dual-Luciferase Assay System was procured from Promega (Madison, WI). E-cadherin-luciferase reporter construct (Hajra, Chen, \& Fearon, 2002) was a gift from Dr. Eric Fearon (University of Michigan). Hypoxia response elements (HRE1 \& 2) in the ZEB2 promoter and ZEB2 binding sites in E-cadherin promoter (E2-box 1 and E2-box 3) were mutated using the Quick-Change Site-Directed Mutagenesis Kit from Stratagene (La Jolla, CA). ZEB2 and ZEB2-NAT promoter constructs (Beltran et al., 2008) were a gift from Dr. Antonio García de Herreros (IMIM, Universitat Pompeu Fabra, Barcelona, Spain). ZEB2 expression construct (Christoffersen, Silahtaroglu, Orom, Kauppinen, \& Lund, 2007) was a gift from Dr. Anders Lund (University of Copenhagen, Copenhagen, Denmark). All other chemicals used were of analytical grade and obtained from Sigma-Aldrich (St. Louis, MO).

\subsection{Hypoxia treatments and surgical procedures}

Wistar rats weighing $190 \pm 10 \mathrm{~g}$, provided by the National Center for Laboratory Animal Sciences (Hyderabad, India), were maintained at $22 \pm 1^{\circ} \mathrm{C}$ and relative humidity of $50 \pm 5 \%$ under a 12-hr light-12-hr dark cycle with food and water ad libitum. Rats were allowed to acclimatize to the animal facility for 1 week. Rats were randomly divided into normoxia and hypoxia cohorts $(n=10)$. Rats in the normoxia group were maintained in a chamber with a fraction of 
inspired oxygen (FIO) of $\sim 0.21$ for 2 weeks. Hypoxia exposure to rats was performed as described earlier (Fan et al., 2016). Briefly, rats were exposed to FIO of 0.1 for $23 \mathrm{hr}$ (hypoxia) and FIO of 0.21 (normoxia) for $1 \mathrm{hr}$ each day and were maintained for 2 weeks (Figure 1a). Rats from both groups were transcardially perfused under anesthesia with saline. Organs were harvested and frozen immediately for further processing or processed for histopathology. The Institutional Animal Ethics Committee of the University of Hyderabad approved animal care and experimental protocols.

\subsection{Estimation of glomerular filtration rate (GFR) and urine parameters}

GFR was estimated in rats $(n=6)$ following 2 weeks of exposure to hypoxia using a commercially available kit (\#FIT-0415; BioPAL, Worcester, MA). Briefly, a single intraperitoneal injection of inulin (25 mg/kg) was given followed by saphenous bleeds at $5,10,30,60$, and $90 \mathrm{~min}$. Plasma was collected, and inulin concentration was estimated using functional immunoassay technology. Plasma clearance of inulin was determined by nonlinear regression using a one-phase

exponential decay formula, and GFR was calculated from inulin clearance as described earlier (O'Brien et al., 2013). Alternatively, spot urine was collected from control and experimental rats and centrifuged at 2,000g for $5 \mathrm{~min}$. Urinary albumin (\#COD11573) and creatinine (\#COD11502) levels were estimated using commercially available assay kits (Biosystems, Barcelona, Spain).

\section{4 | Histological examination and transmission electron microscopy (TEM)}

Renal cortical samples were fixed in $4 \%$ neutral buffered formalin, embedded in paraffin, sectioned $(4 \mu \mathrm{m})$, stained with the periodic acidSchiff (PAS) stain and images were acquired using Olympus 5000 microscope (Olympus Corporation, Waltham, MA) with $\times 40$ magnification. The glomerular tuft area was measured as described earlier (Stojanovic et al., 2012). The extent of glomerulosclerosis index was determined in PAS-stained sections with the scoring system as described earlier (Babelova et al., 2013; Hartner et al., 2001). Briefly, 0: normal glomerulus; 1: mesangial expansion or sclerosis involving up to $25 \%$ of the glomerular tuft; 2 : sclerosis of $25-50 \%$; 3 : sclerosis of $50-75 \%$

(c)

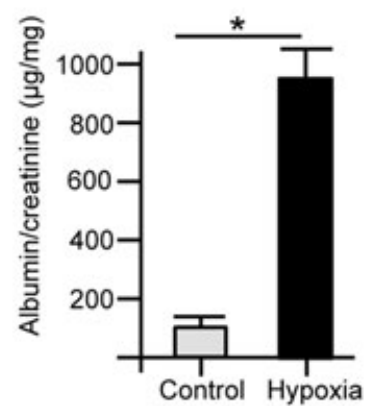

(d)

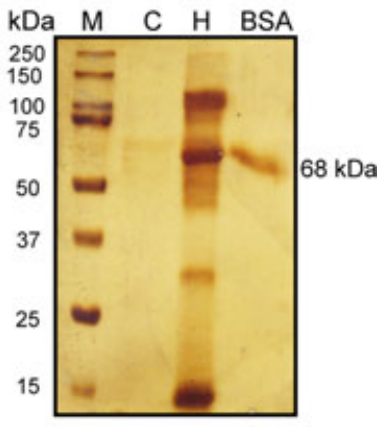

(e)

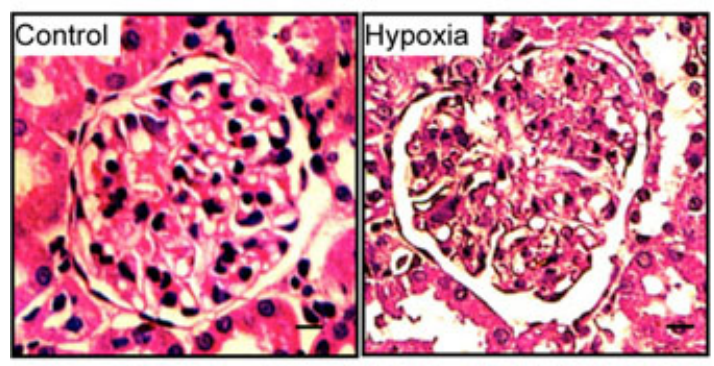

(f)

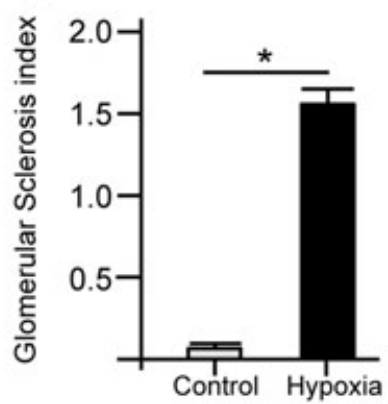

(g)

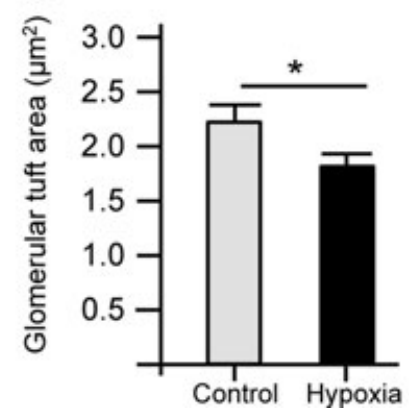

FIGURE 1 Chronic hypoxia alters renal function. (a) Outline of animal experiment. Control rats were maintained at normoxia ( $24 \mathrm{hr} \times 2 \mathrm{weeks}$ ), and experimental rats were exposed to hypoxia ( $23 \mathrm{hr} \times 2$ weeks). After 2 weeks, we assessed renal function tests and histological parameters. (b) Measurement of GFR in rats exposed to hypoxia $\left(n=6,{ }^{*} p<0.01\right.$ ). (c) Estimation of albumin and creatinine levels in rats exposed to hypoxia. The ratio of albumin-creatinine is shown in the form of a bar diagram in each condition $\left(n=6,{ }^{*} p<0.01\right)$. (d) Urine protein analysis in rats exposed to hypoxia. Urine samples were subjected to SDS-PAGE, and band intensities were visualized by silver staining as described in Section 2. M, molecular weight marker (\#1610374; Bio-Rad); C, urine from control rat; $\mathrm{H}$, urine from the rat exposed to hypoxia; and BSA was used as a standard. (e) PAS staining of glomerular regions of rats exposed to hypoxia. (f) Glomerulosclerosis index was assessed as described in Section 2 and (g) measurement of glomerular tuft area in rats exposed to hypoxia. Error bars indicate mean $\pm S E ; n=6$; ${ }^{*} p \leq 0.05$. BSA: bovine serum albumin; GFR: glomerular filtration rate; PAS: periodic acid-Schiff; SDS-PAGE: sodium dodecyl sulfate-polyacrylamide gel electrophoresis 
and/or segmental extracapillary fibrosis or proliferation; and 4: global sclerosis $>75 \%$, global extracapillary fibrosis or complete collapse of the glomerular tuft. For TEM analysis, renal cortex from control and experimental rats were fixed in $2.5 \%$ glutaraldehyde for $24 \mathrm{hr}$. After washing with $1 \times$ phosphate-buffered saline (PBS) for four times, cortical tissues were fixed in $1 \%$ osmium tetroxide for $2 \mathrm{hr}$ and ultrathin sections $(60 \mathrm{~nm})$ were cut and mounted on 200-mesh copper grids. These copper grids were stained on a Leica EM AC20 strainer (Leica Microsystems Inc, Buffalo Grove, IL) with 3\% aqueous uranyl acetate and 3\% lead citrate solution. Images were acquired on a JEM-1400 TEM (Jeol, Peabody, MA) using a Gatan ultrascan CCD camera (Gatan Inc, Pleasanton, $\mathrm{CA}$ ) at $2 \mathrm{~K} \times 2 \mathrm{~K}$ resolution and $120 \mathrm{kV}$.

\section{5 | Isolation of kidney glomeruli}

The glomeruli from the kidneys of control and rats exposed to hypoxia were isolated by a series of stainless sieves (Krtil, Platenik, Kazderova, Tesar, \& Zima, 2007). Briefly, under aseptic conditions, decapsulated rat kidney slices were placed on a sieve with a size of $200 \mu \mathrm{m}$ and gently grounded with a sterile syringe knob and washed with Hank's solution. The filtered kidney lysate was then allowed to pass through decreasing pore size sieve of $150 \mu \mathrm{m}$ and as a final step; the glomeruli were then collected as a suspension on a $75 \mu \mathrm{m}$ sieve. After incubation for $10 \mathrm{~min}$, the suspensions were centrifuged for $5 \mathrm{~min}$ at $1000 \mathrm{rpm}$, then the supernatant was discarded, the purified glomerular precipitations were used for immunoblotting and gene expression studies.

\subsection{Silver staining of urine samples}

Urine samples are collected from control and rats exposed to hypoxia and protein concentrations were estimated using the Bradford assay. Equal amounts of protein were subjected to sodium dodecyl sulfatepolyacrylamide gel electrophoresis (SDS-PAGE), following which gel was placed in fixing solution (50\% methyl alcohol and $10 \%$ glacial acetic acid) for $1 \mathrm{hr}$ at room temperature. Then, the gel was washed with $50 \%$ ethanol and sensitized with sodium thiosulfate for $1 \mathrm{~min}$. After washing with distilled water, the gel was incubated with $0.2 \%$ silver nitrate solution containing $0.037 \mathrm{ml}$ formaldehyde for $30 \mathrm{~min}$. The gel was briefly washed and then developed in $6 \%$ sodium carbonate, containing $0.037 \mathrm{ml}$ formaldehyde solution until protein bands were sufficiently dark. The reaction was terminated by placing the gel in $1 \%$ glacial acetic acid solution and visualized by Bio-Rad image analyzer.

\subsection{Culturing of podocytes and HEK293T cells}

Human podocytes were obtained from the University of Bristol (Bristol, UK) and were cultured as described earlier (Ni, Saleem, \& Mathieson, 2012). Briefly, podocytes were propagated at $33^{\circ} \mathrm{C}$ (permissive conditions) and $5 \% \mathrm{CO}_{2}$ in RPMI-1640 medium containing $10 \%$ fetal bovine serum (FBS), 100 units $/ \mathrm{ml}$ penicillin, $100 \mathrm{mg} / \mathrm{ml}$ streptomycin, and $1 \%$ insulin-transferrin-selenium solution. At $~ 60 \%$ confluence, these cells were transferred to $37^{\circ} \mathrm{C}$ (nonpermissive condition) tissue culture incubator with $5 \% \mathrm{CO}_{2}$ for 6 days to silence the temperature-sensitive tsA58T antigen to allow podocyte differentiation. Differentiation of podocytes was achieved by a growth arrest and the upregulation of cyclin-dependent kinase inhibitors. Cells used for experimentation were from passages 10 to 16 . HEK293T cells were cultured in Dulbecco's modified Eagle's medium supplemented with $10 \%$ FBS, 100 units $/ \mathrm{ml}$ penicillin, $100 \mathrm{mg} / \mathrm{ml}$ streptomycin and incubated at $37^{\circ} \mathrm{C}$ and $5 \% \mathrm{CO}_{2}$. Differentiated podocytes and HEK293T cells were exposed to hypoxia using a modular gas chamber inside $\mathrm{CO}_{2}$ incubator where along with carbon dioxide; nitrogen gas was supplied to reduce the oxygen levels to $1 \%$ for $24 \mathrm{hr}$.

\section{8 | Immunoblotting}

Glomerular lysates from rat kidney cortex, human podocytes, and HEK293T cells were processed for immunoblotting. An equal amount of protein from control and experimental samples was electrophoresed through 10\% SDS-PAGE. After electrophoresis, the proteins were transferred to nitrocellulose membrane by electroblotting. The nitrocellulose membrane was then blocked in $5 \%$ nonfat milk for $1 \mathrm{hr}$, and subsequently probed with the indicated primary (1:1,000 dilution) and secondary antibodies (1:10,000 dilution). Blots were developed using the ECL western blot analysis substrate.

\section{9 | Quantitative real-time polymerase chain reaction (qRT-PCR) analysis}

Total RNA from rat glomeruli, human podocytes, and HEK293T cells was extracted using TRIzol reagent and isolated using an RNA isolation kit. RT-PCR was performed using iQ SYBR Green Supermix (Bio-Rad Laboratories, Hercules, CA). Messenger RNA (mRNA) expression levels were normalized using the expression of $\beta$-actin, and data were analyzed using the comparative threshold cycle method.

\subsection{0 | Immunofluorescence analysis}

Cells cultured on coverslips were fixed with $4 \%$ paraformaldehyde, permeabilized with $0.1 \%$ Triton X-100 and blocked with $3 \%$ bovine serum albumin (BSA) in $1 \times$ PBS. Cells were incubated with primary antibodies for HIF1 $\alpha$ (1:100) and ZEB2 (1:100). HIF1 $\alpha$ and ZEB2 were visualized using Cy5 horse anti-mouse IgG (1:200) and DyLight594 anti-rabbit IgG (1:200), respectively (Vector Laboratories, Burlingame, CA). ProLong gold antifade with 4',6-diamidino-2-phenylindole is used to mount the slides (Thermo Fisher Scientific, Waltham, MA). Images were acquired using a confocal microscope (ZEISS, Thornwood, NY) at $\times 63$ magnification. Similarly, expression of HIF1 $\alpha$ and ZEB2 in glomerular sections of experimental rats were analyzed using a trinocular microscope (Leica, Buffalo Grove, IL). 


\subsection{1 | Cell migration assay}

Both differentiated human podocytes and HEK293T cells were seeded in six-well plates and grown under normal growth conditions to reach $95 \%$ confluence. When the cells are confluent, media was aspirated, and a scratch was made in a sweeping motion using a $10-\mu \mathrm{l}$ sterile pipette tip. Cells were washed with prewarmed media to remove debris from damaged cells. Images of cells were taken following exposure to hypoxia at regular time points to monitor the rate of cell migration into the wound field. The area covered by cells is quantified using Image J software (NIH, Bethesda, MD) and represented as the percentage of area coverage (cell area/region of interest area $\times 100$ ).

\subsection{2 | Short hairpin RNA (shRNA)-mediated ZEB2 knockdown in podocytes}

ZEB2 expression in human podocytes was knockdown as described earlier (Kumar et al., 2010). Lentiviral particles expressing unique ZEB2 shRNA sequences and scrambled shRNA were purchased from Sigma-Aldrich (St. Louis, MO). Podocytes were cultured in a six-well plate under permissive growth conditions for $16 \mathrm{hr}$. The cells were then pretreated with $6 \mu \mathrm{g} / \mathrm{ml}$ polybrene and infected with indicated lentiviral particles. Next day the medium was replaced with fresh medium, and shRNA-infected cells were selected with $1 \mu \mathrm{g} / \mathrm{ml}$ puromycin. Puromycin-resistant cells were induced to differentiate under nonpermissive conditions, and ZEB2 expression was verified in the differentiated cells using qRT-PCR and immunoblotting.

\subsection{3 | Albumin influx assay}

Podocyte permselectivity was assessed using paracellular albumin influx assay as described previously (Kumar et al., 2010). Corning Transwell Collagen-Coated $3.0 \mu \mathrm{m}$ pore polytetrafluoroethylene (PTFE) membrane inserts (\#CLS3494; Sigma-Aldrich, St. Louis, MO) were used in this study. Podocytes were seeded on the transwell insert and placed in a 12-well tissue culture plate, during which transwell insert serves as upper chamber and cell culture well serve as a bottom chamber (Figure 8a). Podocytes were cultured under nonpermissive conditions for 6 days as detailed above in the methods section. These differentiated cells were exposed to hypoxia for $24 \mathrm{hr}$. Subsequently, the cells were washed twice with $1 \times$ PBS containing $\mathrm{CaCl}_{2}$ and $\mathrm{MgCl}_{2}$ to preserve cadherin junctions. Two milliliter of RPMI-1640 medium with $40 \mathrm{mg} / \mathrm{ml}$ BSA was placed in the bottom chamber, and $300 \mu \mathrm{l}$ of RPMI1640 medium (without BSA) was placed in the upper chamber. The cells were incubated at $37^{\circ} \mathrm{C}$, aliquots of medium were collected from the upper chamber at regular intervals, and albumin concentration was measured from these aliquots.

\subsection{4 | Transient transfection and luciferase assay}

HEK293T cells were transfected with a promoter-reporter construct (ZEB2/E-cadherin) and internal control expressing the Renilla luciferase, pRL-TK (Promega). HEK293T cells were transfected using Xfect polymer (DSS Takara Bio, New Delhi, India) as per manufacturer's instructions. After $48 \mathrm{hr}$ of transfection, cells were washed twice with $1 \times$ PBS and harvested in $100 \mu$ of passive lysis buffer. After a brief freeze-thaw cycle, the insoluble debris was removed by centrifugation at $12,000 \mathrm{~g}\left(4^{\circ} \mathrm{C}\right.$ for $5 \mathrm{~min}$ ), and $20 \mu \mathrm{l}$ of supernatant was used for luciferase assay. The activity of the cotransfected $R$. luciferase reporter plasmid was used to normalize transfection efficiency.

\subsection{5 | Chromatin immunoprecipitation (ChIP) assay}

ChIP assay was performed as described previously (Mungamuri, Wang, Manfredi, Gu, \& Aaronson, 2015). HEK293T $\left(2.5 \times 10^{6}\right)$ cells either naïve or exposed to hypoxia were taken for ChIP assay. Crosslinked chromatin from HEK293T cells was sonicated using S220 focused-ultrasonicator (Covaris Inc, Woburn, MA). The whole cell extract was precleared using $60 \mu \mathrm{l}$ of Protein A/G PLUS-agarose beads (Thermo Fisher Scientific, Waltham, MA) and incubated overnight with anti-HIF1 $\alpha$ and control IgG antibodies. One microgram of anti-HIF1 $\alpha$ antibody was used for $100 \mu \mathrm{g}$ of total cell lysate. Protein G agarose beads (Millipore, Saint Charles, MO) preblocked with salmon sperm DNA (60 $\mu$ l) was used for each immunoprecipitation. Ten percent of chromatin from each sample was removed before immunoprecipitation and used as input for PCR amplification. Both immunoprecipitated and input DNA were purified with PCRpurification kit (Qiagen, Valencia, CA). qRT-PCR was performed on this DNA to identify the amount of target sequence. HRE region of the VEGFA promoter was used as a positive control, while the primers that do not amplify the HRE region of the ZEB2 promoter was used as a negative control.

\subsection{6 | Statistical analysis}

SPSS software was used to apply Mann-Whitney and Kruskal-Wallis nonparametric tests to analyze statistical differences between the distributions of two or multiple independent samples, respectively. $p \leq 0.05$ were considered as statistically significant.

\section{3 | RESULTS}

\section{1 | Chronic hypoxia alters glomerular function in vivo}

To assess the impact of chronic hypoxia on renal function, we estimated GFR in vivo (Figure 1b). Rats exposed to hypoxia displayed reduced GFR ( 29\%) compared with control rats (2.42 vs. $3.45 \mathrm{ml}^{-1} \cdot \mathrm{min}^{-1} \cdot \mathrm{kg}^{-1}$ body weight). To confirm the adverse effect of hypoxia on renal function, we also assessed urinary albumin to creatinine ratio. Rats subjected to hypoxia showed $\sim 9$-fold increase in albumin excretion compared with control rats (Figure 1c). Furthermore, urinary fractions collected from rats exposed to hypoxia had a significant amount of protein as assessed by SDS-PAGE and visualized by silver staining (Figure 1d). We measured 
the extent of glomerulosclerosis in PAS-stained glomeruli and found that increased Bowman's space, mild and diffuse glomerulosclerosis (Figure 1e,f) and reduced glomerular tuft area (Figure 1g) in rats exposed to hypoxia. Average glomerular tuft area in rats exposed to hypoxia was $1.8 \mu \mathrm{m}^{2}$ compared with $2.25 \mu \mathrm{m}^{2}$ in control rats. All these data suggested altered glomerular architecture and increased glomerular permeability as evidenced by proteinuria in rats exposed to hypoxia.

\section{2 | Chronic hypoxia-induced podocyte injury in vivo}

To understand the causative factors for proteinuria in rats exposed to hypoxia, we next investigated whether there are any differences in podocyte count and podocyte structure, using immunochemistry and TEM techniques, respectively. We stained glomerular sections for WT1, a putative marker for podocytes, to study the effect of hypoxia on quantification of podocytes (Figure $2 \mathrm{a}$ ). We noticed $\sim 39 \%$ decrease in podocytes number in experimental rats. When compare with podocyte count of $23 \pm 4 /$ glomeruli in control rats, rats exposed to hypoxia had a count of $14 \pm 5$ (Figure 2b). To ascertain the podocyte morphology, we performed TEM analysis and observed foot-processes effacement and disruption of slit-diaphragm from rats exposed to hypoxia (Figure 2c). TEM images also revealed that there are no noticeable changes in glomerular basement membrane and endothelium between control and experimental rats (Figure 2c). All these data suggest podocyte injury in rats exposed to hypoxia.

\section{3 | Chronic hypoxia-induced glomerular ZEB2 expression}

Since, we observed significant morphological and functional changes in the glomerular region, particularly in podocytes of rats exposed to hypoxia, we would like to understand the molecular basis for the observed pathological changes. HIF1 $\alpha$ is a major transcription factor that regulates the adaptive response to hypoxia. Earlier studies suggest that activation of HIF1 $\alpha$ is associated with progressive glomerulonephritis, EMT, fibrosis, and renal failure (Fine \& Norman, 2008). Considering the significance of HIF1 $\alpha$ under hypoxic conditions, we investigated HIF1 $\alpha$ expression in the glomerular region of rats exposed to chronic hypoxia. As expected, we observed elevated expression of HIF1 $\alpha$ in the glomerular region of rats exposed to hypoxia (Figure 3a,b). Further, induction of HIF1 $\alpha$ is correlated with the upregulation of its downstream targets including TG2, VEGFA, MMP2, and MMP9 and decreased expression of podocyte-specific proteins nephrin and podocin (Figure 3a,b). In addition to these canonical HIF1 $\alpha$ targets, we have also analyzed the expression of ZEB2 in the glomerular region of the kidney, since ZEB2 gets upregulated during podocyte injury and elevated ZEB2 expression is associated with podocyte depletion (Chitra et al., 2015; Kumar et al., 2010). Interestingly, we observed an elevated ZEB2 expression in glomerular extracts from rats subjected to hypoxia, as measured at both protein (Figure 3a) and mRNA (Figure 3b) levels. Immunofluorescence data confirms an elevated expression of both HIF1 $\alpha$ and ZEB2 in glomerular regions exposed to hypoxia (Figure 3c). The data suggest that elevated expression of glomerular HIF1 $\alpha$ during hypoxia is associated with increased ZEB2 expression in proteinuric rats.

\subsection{Expression of ZEB2 and ZEB2-NAT is associated with EMT of human podocytes}

ZEB2, which was increased in glomerular extracts of hypoxic rats, is chosen for further analysis owing to its established role in EMT of glomerular podocytes and altering podocyte permeability (Kumar et al., 2010; Wiles, Bell, Thomas, Beckerle, \& Lessnick, 2013). ZEB2 is also involved in the development of the kidneys (Rasouly et al., 2016). ZEB2 genomic locus also expresses ZEB2-NAT in antisense manner and is known to bind complementary region on ZEB2 mRNA and mask the factors that would otherwise induce splicing (Beltran et al., 2008; Wight \& Werner, 2013). Thus, binding of ZEB2-NAT to ZEB2 mRNA prevents its splicing and augments ZEB2 mRNA translation (Kumar et al., 2010). We found elevated expression of ZEB2-NAT in addition to ZEB2 mRNA in glomerular extracts of rats exposed to hypoxia (Figure 4a), indicating dual regulation of ZEB2 mRNA under hypoxic conditions; both at transcriptional (pre-mRNA) and translational (postmRNA) levels. Upon confirming the elevated expression of ZEB2 in hypoxic rats, we assessed the expression of ZEB2 target genes and EMT markers: E-cadherin, a predominant epithelial marker; P-cadherin and ZO-1, constituents of cell-cell junctions; $\mathrm{N}$-cadherin, vimentin, and $\beta$-catenin are mesenchymal markers. ZO-1, E-, and P-cadherin were downregulated, whereas $\mathrm{N}$-cadherin, vimentin, and $\beta$-catenin were upregulated in rat glomerular extracts in response to hypoxia (Figure 4a,b). To validate the observations found in rats also holds true in humans, we analyzed the expression of the same set of genes in Nakagawa chronic kidney disease (CKD) data set available at Nephroseq (https://nephroseq.org). This analysis revealed coexpression of HIF1 $\alpha$, ZEB2, $\beta$-catenin in CKD data set (Figure 4c). It should be noted that elevated expression of HIF1 $\alpha$ is also associated with loss of nephrin and podocin in CKD patients (Figure 4c). Thus, human renal genome-wide gene expression analysis also suggests a direct association of elevated HIF1 $\alpha$ levels with loss of podocyte-specific gene expression.

\section{5 | Hypoxia induces HIF1 $\alpha$ and ZEB2 expression in podocytes in vitro}

Glomerular podocytes are instrumental to ensure glomerular permselectivity (Anil Kumar et al., 2014). It was reported that podocytes undergo EMT and such a glomerular injury with reduced podocyte count was associated with proteinuria (Chitra et al., 2015; Nishad, Nakuluri, Motrapu, \& Pasupulati, 2017). To investigate whether the observed proteinuria and decreased podocyte count in rats exposed to hypoxia were owing to podocyte injury, we then investigated the effect of hypoxia on human immortalized podocytes. As anticipated, upon exposure to hypoxia, we noticed the accumulation of HIF1 $\alpha$ in podocytes (Figure 5a,b). In addition, we also observed elevated expression of ZEB2 and N-cadherin along with decreased expression of $\mathrm{E}$ - and P-cadherins (Figure $5 \mathrm{a}, \mathrm{b}$ ). We observed induction of 
ZEB2-NAT in podocytes exposed to hypoxia (Figure $5 \mathrm{~b}$ ), similar to the observations made in rat glomerular extracts. Next, we investigated if there is any colocalization of HIF1 $\alpha$ and ZEB2 in the podocytes upon exposure to hypoxia (Figure $5 \mathrm{c}$ ). In podocytes cultured under normoxia, we observed very low levels of HIF1 $\alpha$. In cells exposed to hypoxia HIF1 $\alpha$ levels were elevated and predominantly found in the nucleus (Figure 5c). ZEB2 was also increased in podocytes during hypoxic conditions (Figure $5 c$ ). Together, these data obtained from podocytes (Figure $5 \mathrm{c}$ ) and glomerular sections (Figure 3c) suggest that both HIF1 $\alpha$ and ZEB2 are localized to glomerular podocytes during hypoxic conditions. Elevated expression of ZEB2 in podocytes was associated with cadherin switch as evidenced by attenuation of epithelial cadherins ( $\mathrm{E}$ - and P-cadherins) and acquisition of mesenchymal cadherin ( $\mathrm{N}$-cadherin; Figure $5 \mathrm{a}, \mathrm{b}$ ). To ascertain the elevated expression of ZEB2 during hypoxia ensures cadherin switch, we assessed cadherin expression in HEK293T cells that ectopically express ZEB2 (Figure 5d). Ectopic expression of ZEB2 resulted in the loss of $E$ - and P-cadherins and increase in $\mathrm{N}$-cadherin (Figure $5 \mathrm{~d}$ ). Cadherin switch that is associated with EMT imparts enhanced migratory ability to cells (Wheelock, Shintani, Maeda, Fukumoto, \& Johnson, 2008). Therefore, we next assessed the migratory properties of cells naïve to hypoxia or exposed to hypoxia. Both, podocytes and HEK293T cells exposed to hypoxia showed enhanced migratory properties as assessed by wound healing (a)

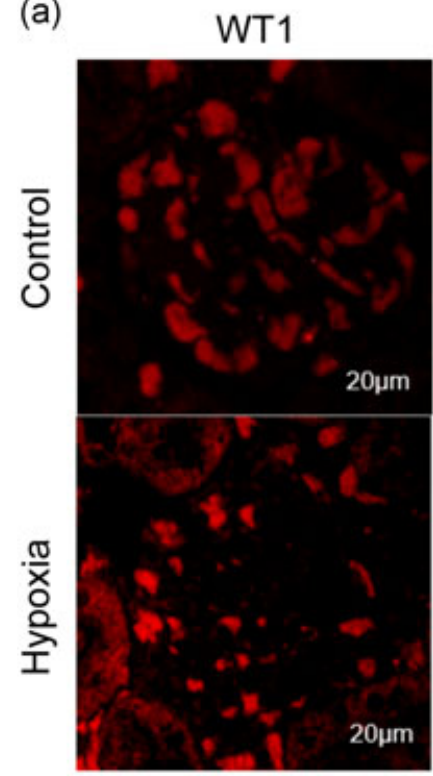

DAPI

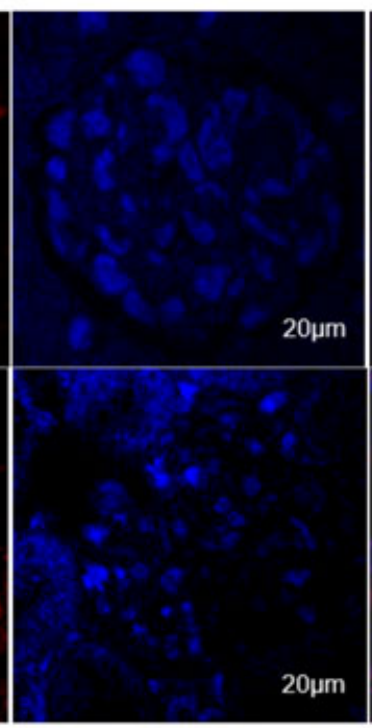

Merge

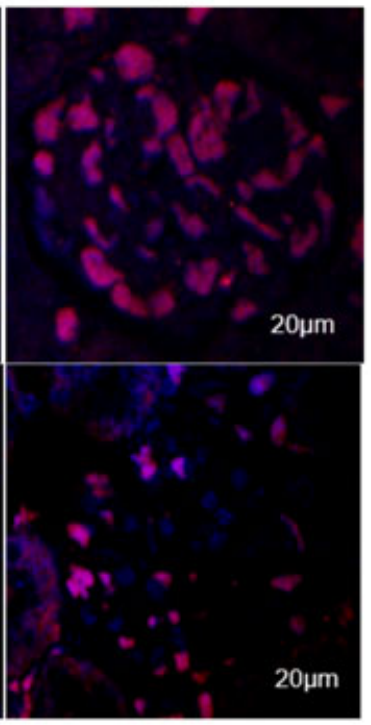

(b)

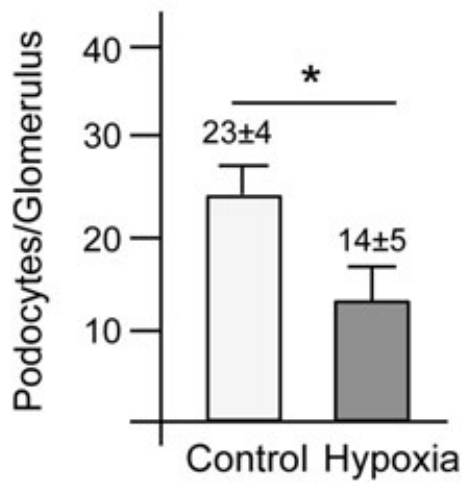

(c)

Control

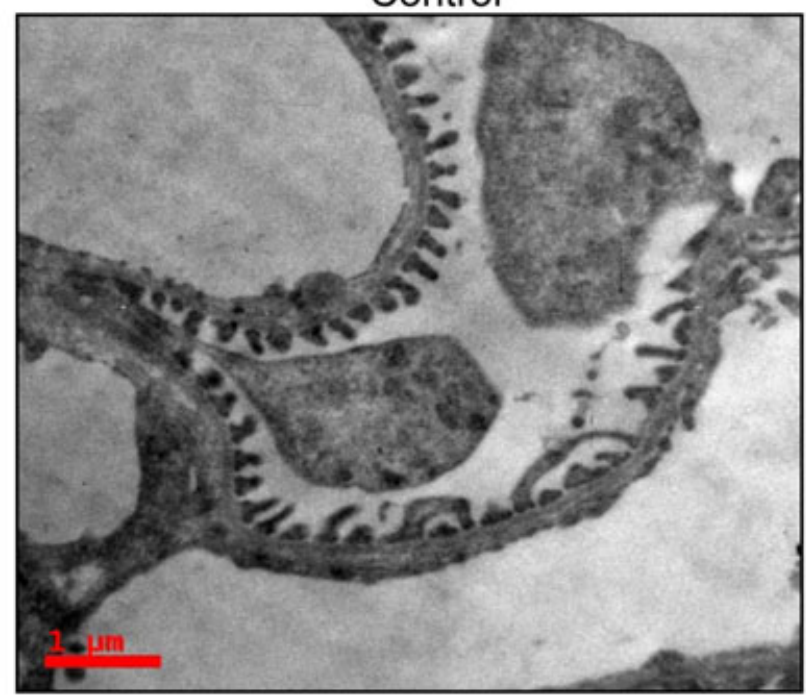

\section{Hypoxia}

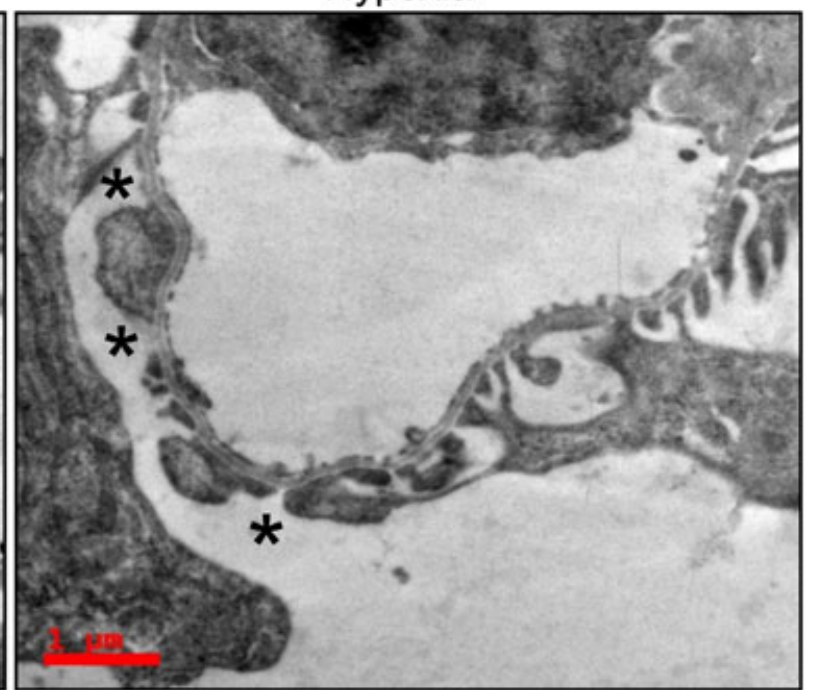

FIGURE 2 Hypoxia induces podocyte injury. (a) WT1 staining of glomerulus from rats exposed to hypoxia. (b) Quantification of podocyte number in these rats. Error bars indicate mean $\pm S E ; n=6 ;{ }^{*} p \leq 0.05$. (c) TEM images of podocytes from rats exposed to hypoxia. These are a representative image out of 12 sections from each condition. Podocyte foot-processes effacement and disruption of slit-diaphragm can be noticed from rats exposed to hypoxia. *Significant alterations in the morphology of podocyte foot-processes occurred in rats exposed to hypoxia. DAPI: 4',6-diamidino-2-phenylindole; TEM: transmission electron microscopy 
assay (Figure 6a-c), a typical feature of cells that had undergone a phenotypic switch from epithelial to mesenchymal state. To verify, whether the enhanced migratory properties of these cells are indeed due to elevated ZEB2 expression, we assessed migratory properties of HEK293T cells that ectopically express ZEB2. Cells overexpressing ZEB2 (Figure 6d) showed enhanced migratory properties upon exposure to hypoxia (Figure 6e). Taken together these data suggest the existence of a strong positive correlation of HIF1 $\alpha$ and ZEB2 expression in the podocytes during hypoxia and induction of ZEB2 ensures podocytes acquire mesenchymal phenotype.

\subsection{HIF1 $\alpha$ increases ZEB2 and ZEB2-NAT promoter activity}

To investigate the effect of HIF1 $\alpha$ on ZEB2 promoter activity, we transiently transfected HEK293T cells with ZEB2 promoterluciferase construct and measured luciferase activity in the cell lysates with and without hypoxia treatment. We observed an increase in ZEB2 promoter activity in cells exposed to hypoxia (Figure 7a). Similarly, ZEB2-NAT promoter activity was also increased with hypoxia (Figure 7a). To verify the specificity of the effect of HIF1 $\alpha$ on ZEB2 promoter activity, we tested the effect of hypoxia on the promoter of an unrelated gene, growth hormone receptor (GHR). Hypoxia had no effect on GHR promoter activity (Figure 7a).

HIF1 $\alpha$ binds to HRE and activates the transcriptional activity of target genes. HRE possess two core sequences; 5'-CACGT-3' and 5'-GCGTG-3' (Katoh \& Katoh, 2009; Wang \& Semenza, 1993). HREs are located at $+104 /+108$, and $+176 /+180$ region in $5^{\prime}$ UTR of ZEB2 gene (Figure 7b). To ascertain the occupancy of HIF1 $\alpha$ on the ZEB2 genomic loci, we engineered ZEB2 promoter-luciferase construct with a mutation in the HREs that abrogate HIF1 $\alpha$ binding to ZEB2 promoter and tested the effect of hypoxia on the activity of this mutant luciferase reporter construct. Site-directed mutations in HREs of ZEB2 promoter failed to elicit ZEB2 promoter-reporter activity in cells exposed to hypoxia (Figure $7 b$ ). These results suggest (a)

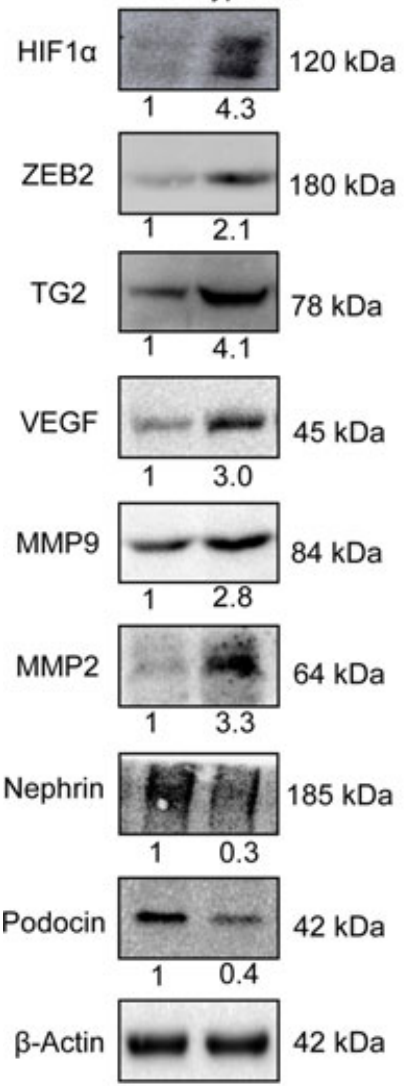

(b)

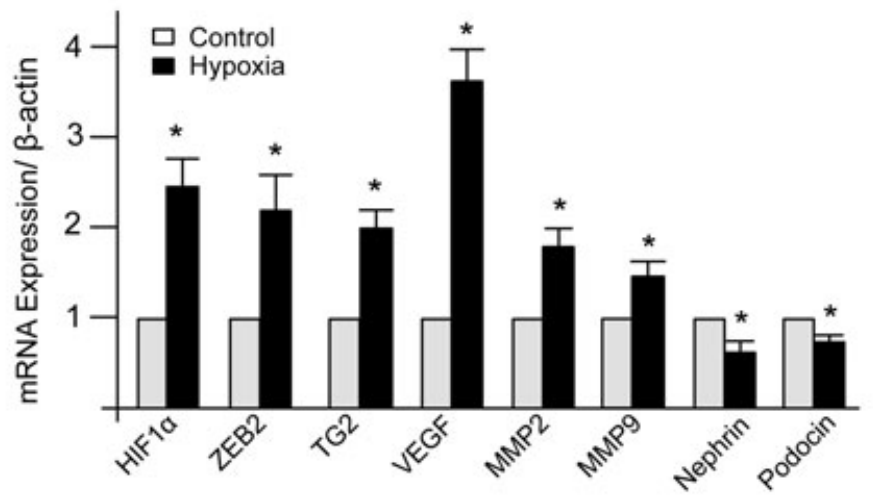

(c)
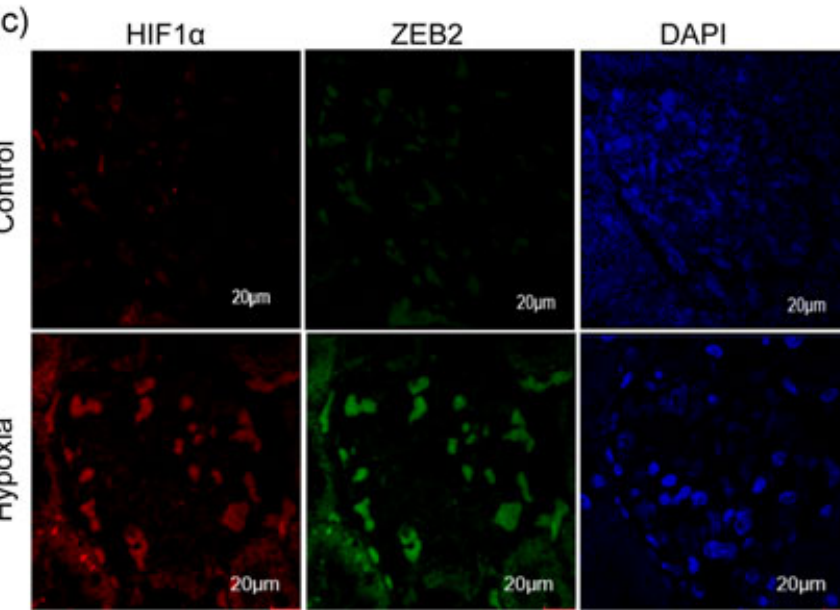

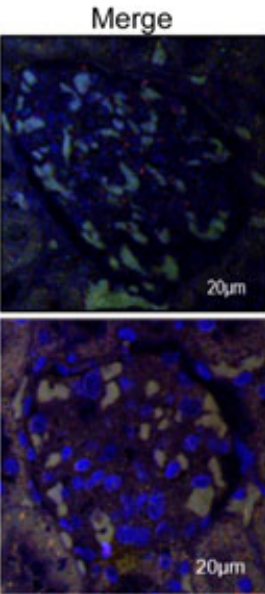

FIGURE 3 Elevated expression of HIF1 $\alpha$ in rat glomerular extracts. Expression of HIF1 $\alpha$ and its targets in rat glomerular extracts is shown as analyzed by immunoblotting (a) and qRT-PCR (b). Densitometric analysis of western blots $(n=3-4)$ was normalized to $\beta$-actin expression and is shown below each blot (a). (b) Error bars indicate mean $\pm S E ; n=4 ;{ }^{*} p \leq 0.05$. (c) Immunofluorescence analysis of HIF1 $\alpha$ and ZEB2 in the rat glomeruli. Images were captured at $\times 63$ using a Leica trinocular microscope. HIF1 $\alpha$ : hypoxia-inducible factor-1 $\alpha$; qRT-PCR: quantitative real-time polymerase chain reaction; TG2: tissue transglutaminase; ZEB2: zinc finger E-box binding homeobox 2; VEGF: vascular endothelial growth factor; MMP: matrix metallopeptidase 


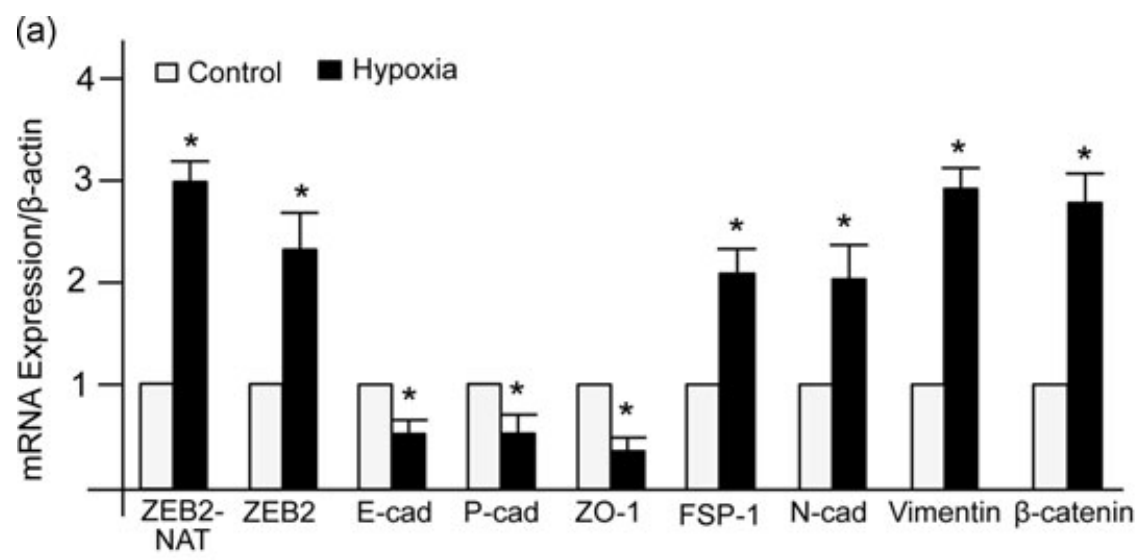

(b)
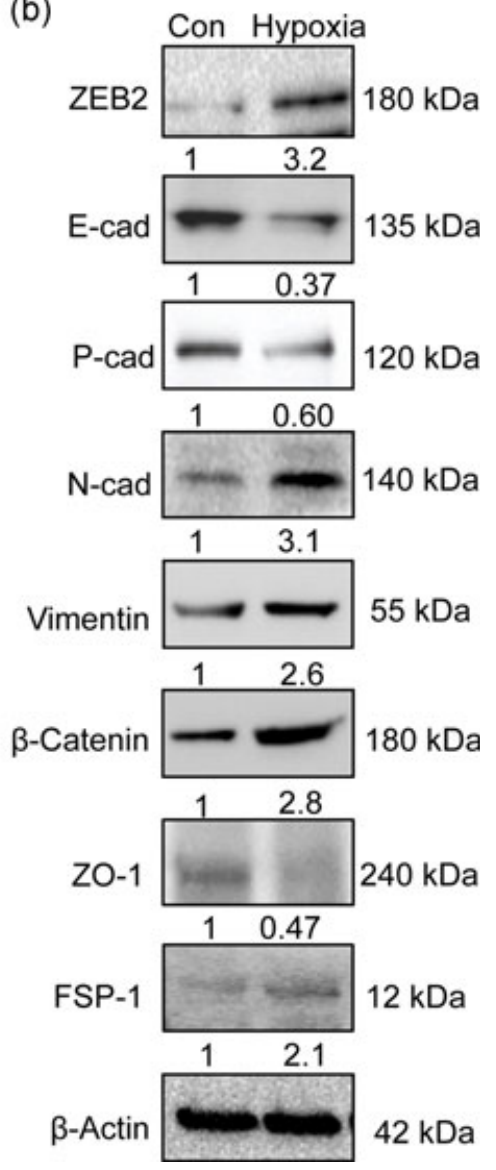

(c)

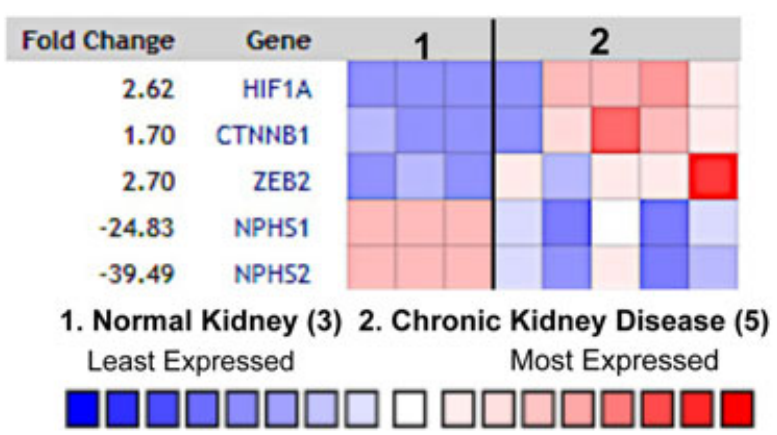

$42 \mathrm{kDa}$

FIGURE 4 Elevated expression of EMT markers in rat glomerular extracts. qRT-PCR (a) and immunoblotting (b) showing the expression of ZEB2, ZEB2-NAT, and EMT markers. (a) Error bars indicate mean $\pm S E ; n=4 ;{ }^{*} p \leq 0.05$. (b) Densitometric analysis of ZEB2 targets normalized for $\beta$-actin expression is shown below each blot $(n=3-4)$. (c) Nephroseq (The Regents of The University of Michigan, Ann Arbor, MI) analysis comparing HIF1A (HIF1alpha), CTNNB1 (beta-catenin), ZEB2, NPHS1 (nephrin), and NPHS2 (podocin) expression levels from patients with CKD versus control. Expression of HIF1 $\alpha$ (2.62-fold), $\beta$-catenin (1.70-fold) and ZEB2 (2.7-fold) are increased; nephrin and podocin expression is decreased several fold among patients with CKD. CKD: chronic kidney disease; EMT: epithelial-mesenchymal transition; mRNA: messenger RNA; NAT: natural antisense transcript; qRT-PCR: quantitative real-time polymerase chain reaction; ZEB2: zinc finger E-box binding homeobox 2; ZO-1: zonula occludens-1; FSP-1: fibroblast-specific protein-1

that functional HREs present on the ZEB2 promoter are essential for ZEB2 expression under hypoxic conditions.

To assess the elevated expression of ZEB2 during hypoxia is due to HIF1 $\alpha$ occupancy on the ZEB2 promoter, we next performed ChIP assay. ChIP assay confirmed that HIF1 $\alpha$ occupies ZEB2 promoter in vivo at the HREs in response to Hypoxia (Figure 7c). The specificity of these associations was verified by demonstrating a lack of binding to a region adjacent to HREs in the ZEB2 promoter. We utilized the interaction of HIF1 $\alpha$ with the promoter region of VEGFA, which serves as a positive control (Forsythe et al., 1996) for the experimental conditions as VEGFA promoter has HRE (Figure 7c). As we observed that ZEB2-NAT was also expressed under hypoxic condition, we performed promoter analysis of ZEB2-NAT for the presence of HREs. We observed HRE sequence ("GCGTG") at two regions of ZEB2-NAT promoter $+1145 /+1149$ and +2945/+2949 Together, this data suggests that HIF1 $\alpha$ elicits ZEB2 protein accumulation by inducing both ZEB2 mRNA and ZEB2-NAT expression wherein ZEB2-NAT augments the expression of ZEB2 mRNA.

\section{7 | ZEB2 knockdown prevents HIF1 $\alpha$-dependent decrease in E-cadherin in podocytes}

We next investigated whether ZEB2 plays an essential role in the HIF1 $\alpha$-mediated podocyte injury. For this, we utilized podocytes in which ZEB2 expression was knocked down using shRNA-mediated approach. After having confirmed the efficacy and specificity of knockdown (Figure 7d), these cells were exposed to hypoxia, and the expression of E-cadherin was measured by immunoblotting. In podocytes transduced with lentivirus expressing ZEB2 shRNA, exposure to hypoxia failed to elicit the decrease in E-cadherin expression (Figure 7e). These results suggest that ZEB2 is essential for transducing the effect of HIF1 $\alpha$ on E-cadherin expression in the podocytes. Further, we investigated the temporal association of HIF1 $\alpha$-induced ZEB2 on E-cadherin expression using E-cadherin promoter construct. E-cadherin promoter has two E2 boxes (E2-box 1: "CAGGTG" \& E2-box 3: "CACCTG") wherein ZEB2 interacts and transduces the repressive action on E-cadherin 
(a)

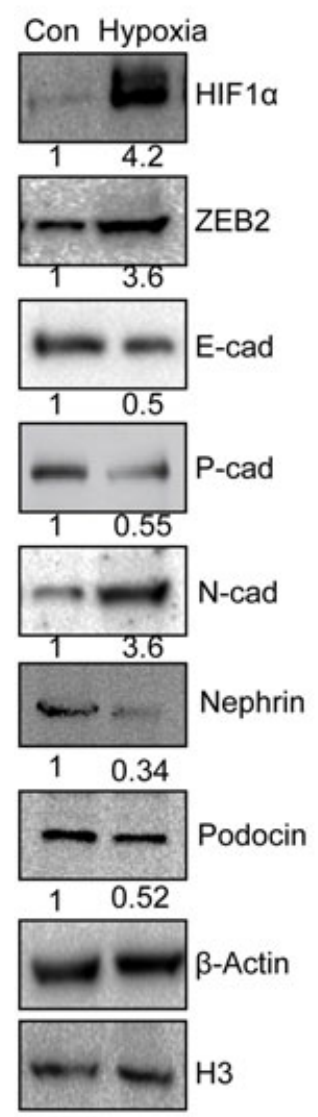

(b)

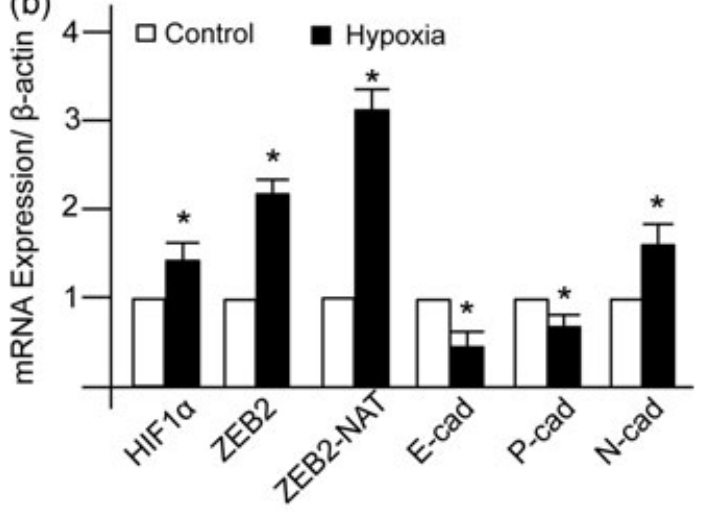

(c)

HIF1a

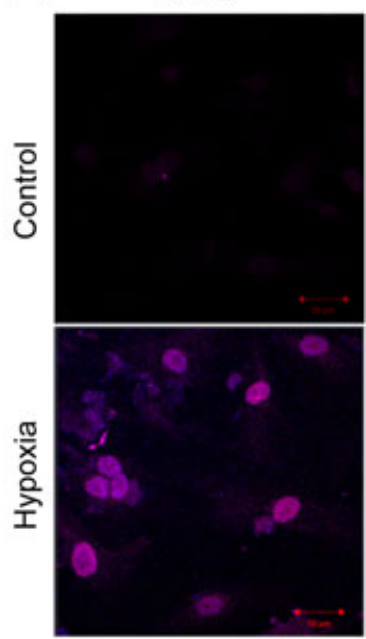

(d)

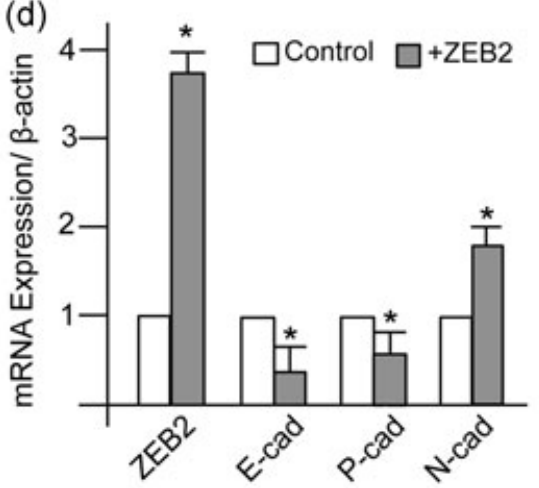

DAPI

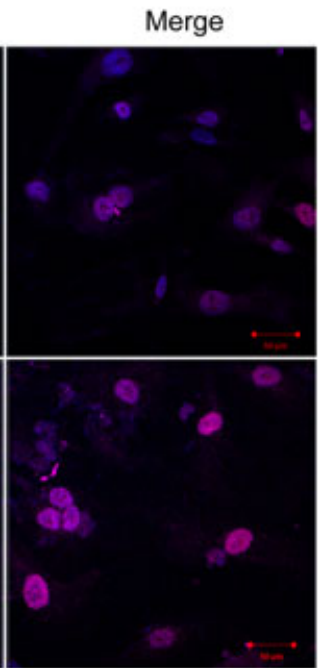

FIGURE 5 Hypoxia induces HIF1 $\alpha$, ZEB2 and EMT markers in human podocytes. Immunoblotting (a) and qRT-PCR (b) analysis of HIF1 $\alpha$, ZEB2, and its targets in human podocytes in response to hypoxia. (a) Densitometric analysis of protein expression was normalized for $\beta$-actin expression and is shown below each blot $(n=3-4)$. (b) The data presented as mean $\pm S E ; n=4-6 ;{ }^{*} p<0.05$. (c) Immunofluorescence images showing elevated expression and colocalization of HIF1 $\alpha$ and ZEB2 in podocytes exposed to hypoxia. Images were acquired using ZEISS confocal microscope. (d) qRT-PCR analysis of cadherins in HEK293T cells that ectopically express ZEB2; mean $\pm S E ; n=4-6 ;{ }^{*} p \leq 0.05$. HIF1 $\alpha$ : hypoxia-inducible factor-1 $\alpha$; qRT-PCR: quantitative real-time polymerase chain reaction; ZEB2: zinc finger E-box binding homeobox 2

expression. The specific effect of HIF1 $\alpha$-induced ZEB2 on E-cadherin expression was analyzed using E-cadherin construct in which ZEB2 binding sites (E2 boxes) were mutated (E-cad-E2M). While hypoxia treatment decreased the activity of E-cad WT promoter activity, it had no effect on E-cad-E2M (Figure 7f), suggesting that the observed loss of E-cadherin expression in hypoxia conditions is due to ZEB2 overexpression.

\section{8 | Essential role of ZEB2 in eliciting the effect of HIF1 $\alpha$ on the permeability of podocytes}

To assess the functional consequence of hypoxia on podocyte function, we next examined the podocyte permeability employing paracellular albumin influx assay. This assay measures the rate of albumin flux across the podocyte monolayer plated on semipermeable PTFE membrane inserts (Figure 8a). After $24 \mathrm{hr}$ of exposure to hypoxia, albumin was placed in the bottom chamber of transwell, and the influx of albumin across the podocyte monolayer was measured at 1 and $4 \mathrm{hr}$ intervals. Increased albumin influx was noticed across the podocyte monolayer that was exposed to hypoxia (Figure $8 \mathrm{~b}$ ). These results revealed a hypoxia-dependent increase in albumin influx across the podocyte monolayer. To delineate the role of HIF $1 \alpha$ on the permeability of the podocyte monolayer, we next investigated the effect of HIF $1 \alpha$ in podocytes in which ZEB2 expression was knocked down. Knocking down of ZEB2 expression resulted in blunting the ability of hypoxia to elicit an increase in the albumin influx across podocyte monolayer (Figure $8 \mathrm{~b}$ ). These results suggest that ZEB2 is necessary for transducing the effect of hypoxia on podocyte permeability. All these data confirm that ZEB2 is the key player in eliciting the proteinuric effect of elevated HIF1 $\alpha$ expression in the podocytes during hypoxic conditions, wherein hypoxia induces HIF1 $\alpha$ expression, which recognizes and binds to HREs on the ZEB2 promoter and transactivates its expression. ZEB2, in turn, regulates the expression of EMT genes, and repression of slitdiaphragm components resulting in increased permeability. 
(a)
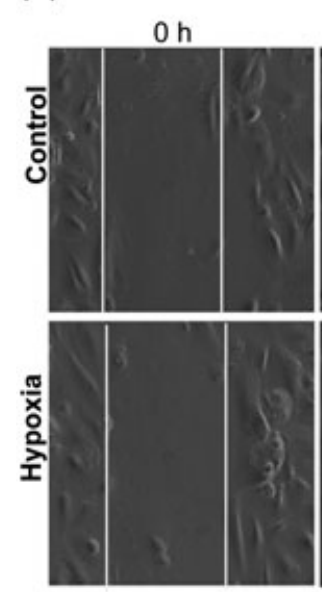

(c)

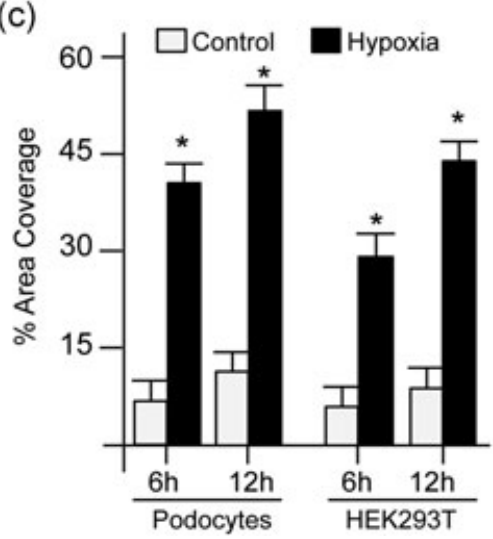

(b)
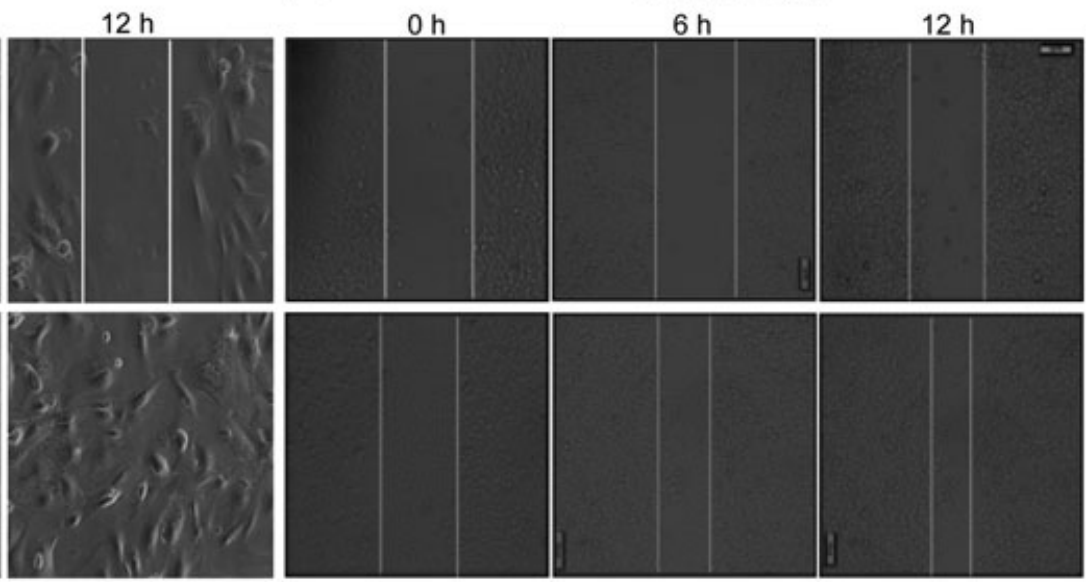

(d)

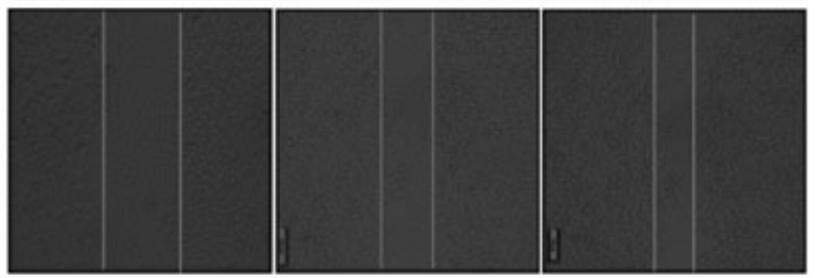

(e)

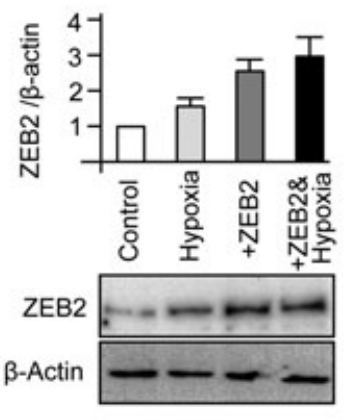

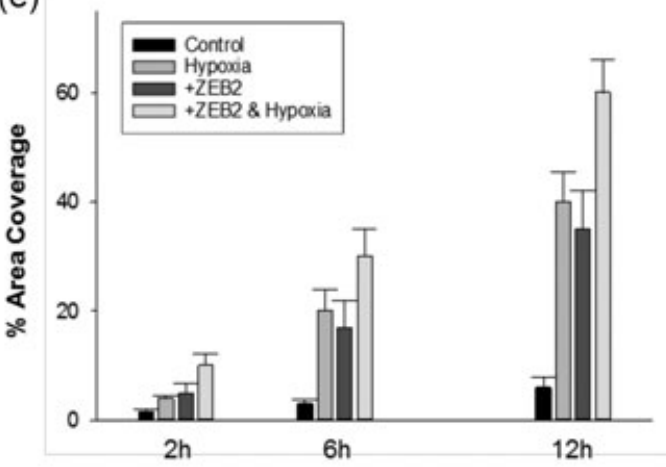

Duration of Exposure to Hypoxia

FIGURE 6 Elevated expression of ZEB2 induces enhanced migration of podocytes. Wound healing assay showing the enhanced migration of human podocytes (a) and HEK293T cells (b) exposed to hypoxia for varying periods of time. (c) Cell migration is monitored and presented as percentage of area covered. (d) Western blot analysis of ZEB2 in HEK293T cells overexpressing ZEB2 and quantification of band intensities. (e) Wound healing assay of HEK293T cells (control and ZEB2 overexpressing) and cell migration is monitored and presented as \% area covered. (c-e) The data are presented as mean $\pm S E ; n=4-6 ;{ }^{*} p \leq 0.05$. ZEB2: zinc finger E-box binding homeobox 2

\section{4 | DISCUSSION}

Prevalence of glomerular dysfunction is prominent in clinical conditions and adverse environmental conditions wherein hypoxia prevails. Nevertheless, the pathological events that connect hypoxia to glomerular dysfunction remain largely unknown. We took up the current investigation to understand the pathophysiological basis of the effects of chronic hypoxia on glomerular podocytes. Our study revealed that in rats exposed to chronic hypoxia, HIF1 $\alpha$ is elevated in glomerular regions. Our results indicate a novel target of HIF1 $\alpha$ is the induction of ZEB2. This increase in ZEB2 expression is due to the upregulation of ZEB2 mRNA and the induction of NAT of the ZEB2 gene. We demonstrated that HIF1 $\alpha$-dependent induction of ZEB2 in the podocytes results in decreased expression of $\mathrm{P}$ - and $\mathrm{E}$-cadherin. P-cadherin is a component of the podocyte slit-diaphragm (Reiser, Kriz, Kretzler, \& Mundel, 2000), and E-cadherin anchors epithelial junctions and participates in EMT (Batlle et al., 2000). Our studies also establish that accumulation of HIF1 $\alpha$ increases permeability of a podocyte monolayer, and this effect is a consequence of HIF1 $\alpha$ - dependent induction of ZEB2. A hyperactive HIF1 $\alpha$-ZEB2 axis is implicated in the altered podocyte biology, both by initiating EMT and altered permeability to the slit-diaphragm (Figure 8c); and our results provide a molecular basis for the role of hypoxia in the pathogenesis of glomerular diseases.

The hypoxic condition results in decreased arterial oxygen pressure that could affect multiple organs including the kidney. Arterial oxygen pressure is maintained at relatively stable levels by a unique and complex functional interplay between renal blood flow, GFR, oxygen consumption, and arteriovenous oxygen shunting (Haase, 2013; Nangaku, 2011). The fragility of this interplay makes the kidney vulnerable to hypoxic injury (Haase, 2013). Renal cells have evolved a variety of molecular mechanisms to adapt to hypoxic conditions that prevail during development, under extreme physiological and pathological conditions. HIF1 $\alpha$ is a predominant transcription factor involved in the control of hypoxia and oxidative stress responses. It is noteworthy that intrarenal hypoxia is a proposed pathway to renal dysfunction in conditions such as diabetes and hypertension (Franzen, Pihl, Khan, Gustafsson, \& Palm, 2016). 
(a)

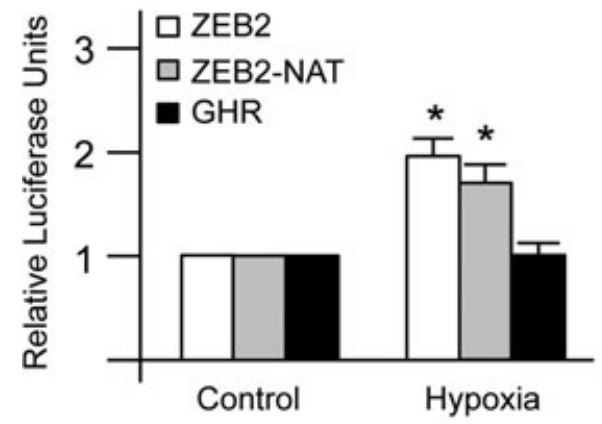

(c)

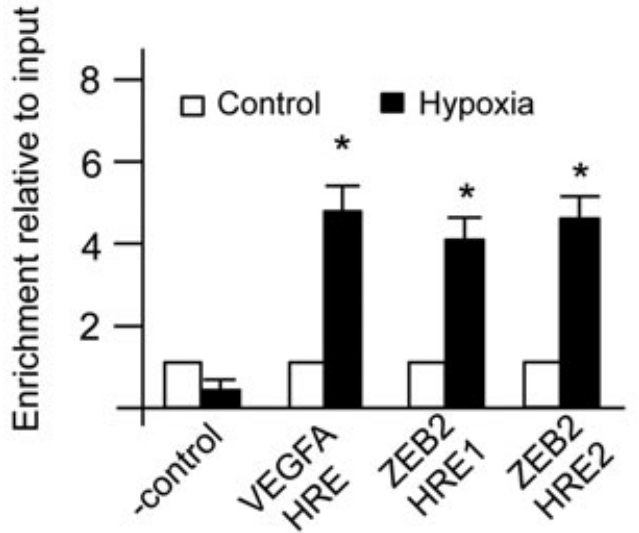

(b)

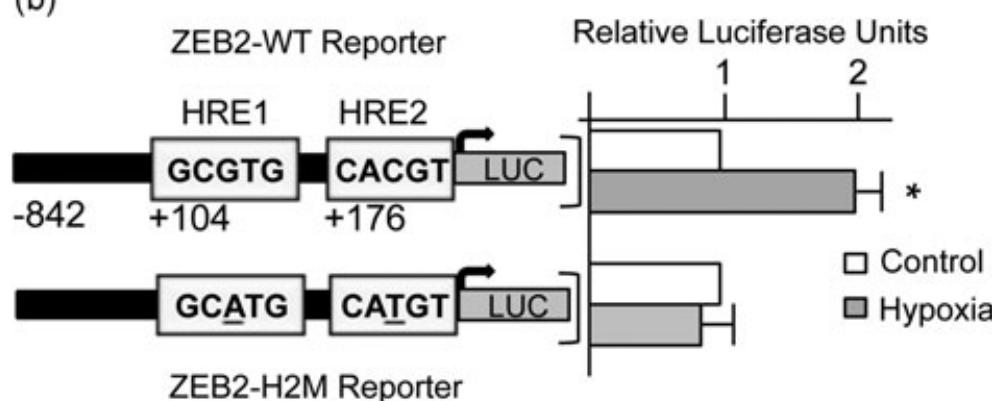

(e)

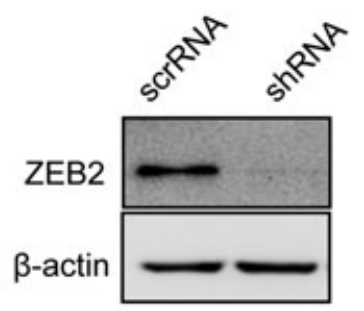

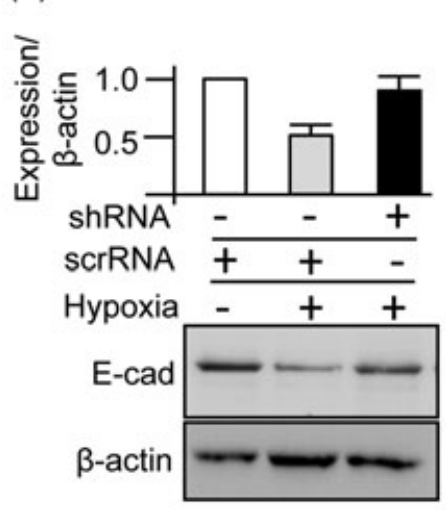

(f)

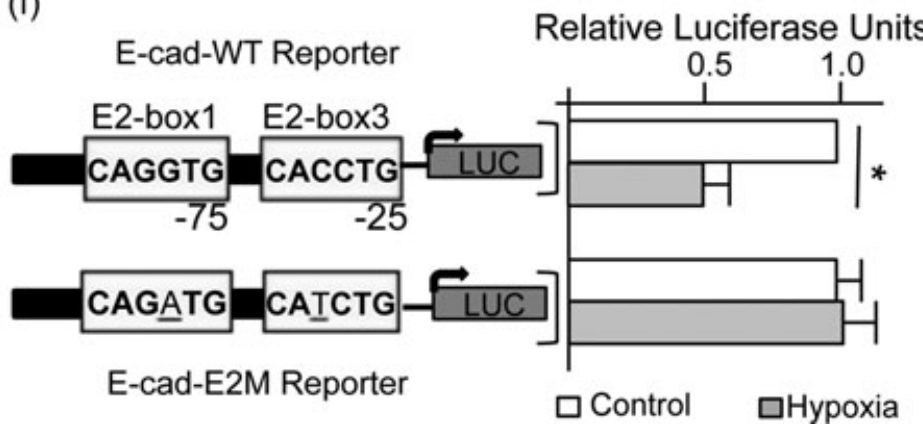

FIGURE 7 Hypoxia induces HIF1 $\alpha$-ZEB2 axis. (a) Luciferase assay in HEK293T cells transiently transfected with indicated constructs and exposed to hypoxia for $24 \mathrm{hr}$. Renilla luciferase was used as an internal control to normalize transfection efficiency. (b) Left panel: Schematic representation of ZEB2 5'UTR showing localization of HREs and point mutations in HREs. Right panel: HEK293T cells were transiently transfected with ZEB2 promoter-luciferase (ZEB2-WT) or ZEB2 promoter-luciferase construct with point mutations in the both HREs (ZEB2-H2M) and exposed to hypoxia for $24 \mathrm{hr}$. R. luciferase is used to normalize transfection efficiency. The normalized luciferase activity for each individual construct exposed to normoxia is designated as 1-fold. (c) ChIP analysis with chromatin fractions from HEK293T cells exposed to hypoxia was performed as described. Input DNA and DNA from each of the immunoprecipitated samples were PCR amplified for HRE in both ZEB2 promoter and VEGFA promoter. (d) Western blot analysis of ZEB2 showing the efficiency of ZEB2 shRNA. (e) Western blot analysis showing E-cadherin expression in human podocytes transduced with a lentiviral construct expressing ZEB2 shRNA with and without hypoxia. (f) Left panel: Schematic representation of E-cadherin promoter region showing localization of E2-box 1 and E2-box 3 and point mutations in both E2 boxes. Right panel: HEK293T cells were transiently transfected with E-cadherin promoter-luciferase (E-cad-WT) or E-cadherin promoter-luciferase construct with point mutations in the both E2-box 1 and 3 (E-cad-E2M) and exposed to hypoxia for $24 \mathrm{hr}$. $R$. luciferase is used to normalize transfection efficiency. The normalized luciferase activity for each individual construct exposed to normoxia is designated as 1 fold. (a-c, e,f) The data are presented as mean $\pm S E ; n=4-6$; ${ }^{*} p \leq 0.05$. Chlp: chromatin immunoprecipitation; HIF1 $\alpha$ : hypoxia-inducible factor-1 $\alpha$; GHR: growth hormone receptor; HREs: hypoxia response elements; PCR: polymerase chain reaction; shRNA: short hairpin RNA; ZEB2: zinc finger E-box binding homeobox 2; VEGFA: vascular endothelial growth factor A

Proteinuria is a predominant manifestation of glomerular dysfunction, and it refers to the alterations particularly in GFB that otherwise offers permselectivity and prevents loss of protein in the urine. Therefore, we investigated the effect of hypoxia in renal cortex, the region where glomeruli are concentrated, and in podocytes that are instrumental to ensure permselectivity. It is interesting to note that HIF1 $\alpha$ levels have been reported to be elevated in many organs including the liver, brain, and kidney in 
response to hypoxia and influence target gene expression (Majmundar, Wong, \& Simon, 2010). Similarly, in our model HIF1 $\alpha$ levels were significantly upregulated in the kidney compared to liver, and brain.

Our results establish that hypoxia-induced accumulation of HIF1 $\alpha$ stimulates the expression of ZEB2. ZEB2, also known as Smad-interacting protein-1 (SIP1) and ZFHX1B, belongs to the ZEB protein family. ZEB2 acts as a transcriptional repressor in both C-terminal binding protein (CTBP) dependent and independent manner (van Grunsven et al., 2003). The expression of ZEB2, a critical transcriptional factor that orchestrates EMT phenomenon is regulated by cis-NAT. We observed expression of ZEB2-NAT in podocytes exposed to hypoxia. Since ZEB2 has many potential targets including crucial proteins of tight junctions, adherence junctions and gap junctions (Vandewalle et al., 2005), the increase in ZEB2 could have pleiotropic effects on the podocyte biology

(a)

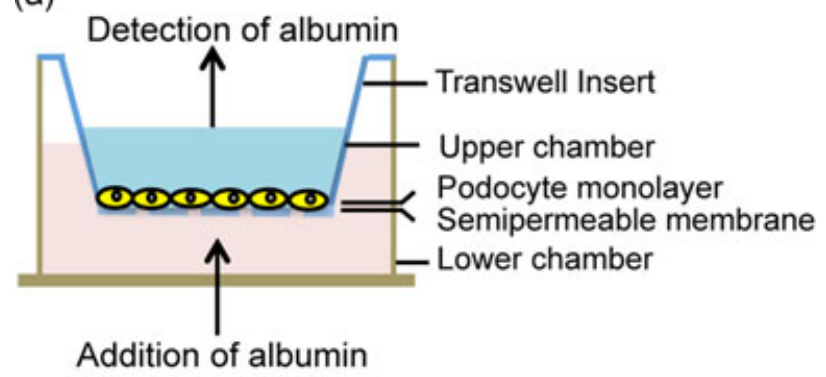

during hypoxia. E-cadherin is a canonical target for transcriptional repression by ZEB2. E-cadherin exists in cytoplasmic vesicles in podocytes in culture (Reiser et al., 2000). The role of E-cadherin in EMT phenomenon is well established, wherein E-cadherin expression is downregulated during the transition of cells from epithelial to mesenchymal phenotype (Palacios, Tushir, Fujita, \& D'Souza-Schorey, 2005). P-cadherin, another bonafide target of ZEB2, belongs to the cadherin superfamily and is a component of the slit-diaphragm apparatus. Decreased expression of P-cadherin is implicated in the pathogenesis of proteinuria and glomerulosclerosis (Boini et al., 2012; Xu et al., 2005). P-cadherin mediates cell-cell adhesion between adjacent podocyte foot processes and also serves as the basic scaffold for the slit-diaphragm. It is noteworthy that the key findings of the current study, HIF1 $\alpha$-dependent increase in ZEB2 and the resultant decrease in E- and P-cadherins, were observed in both

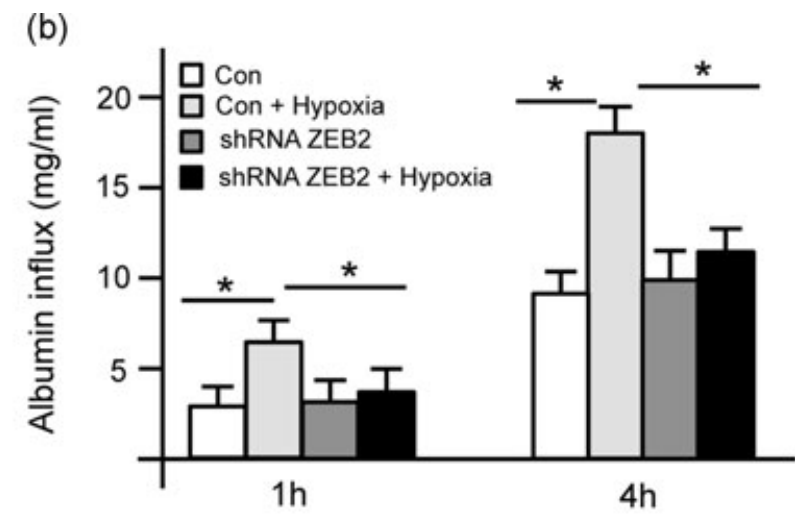

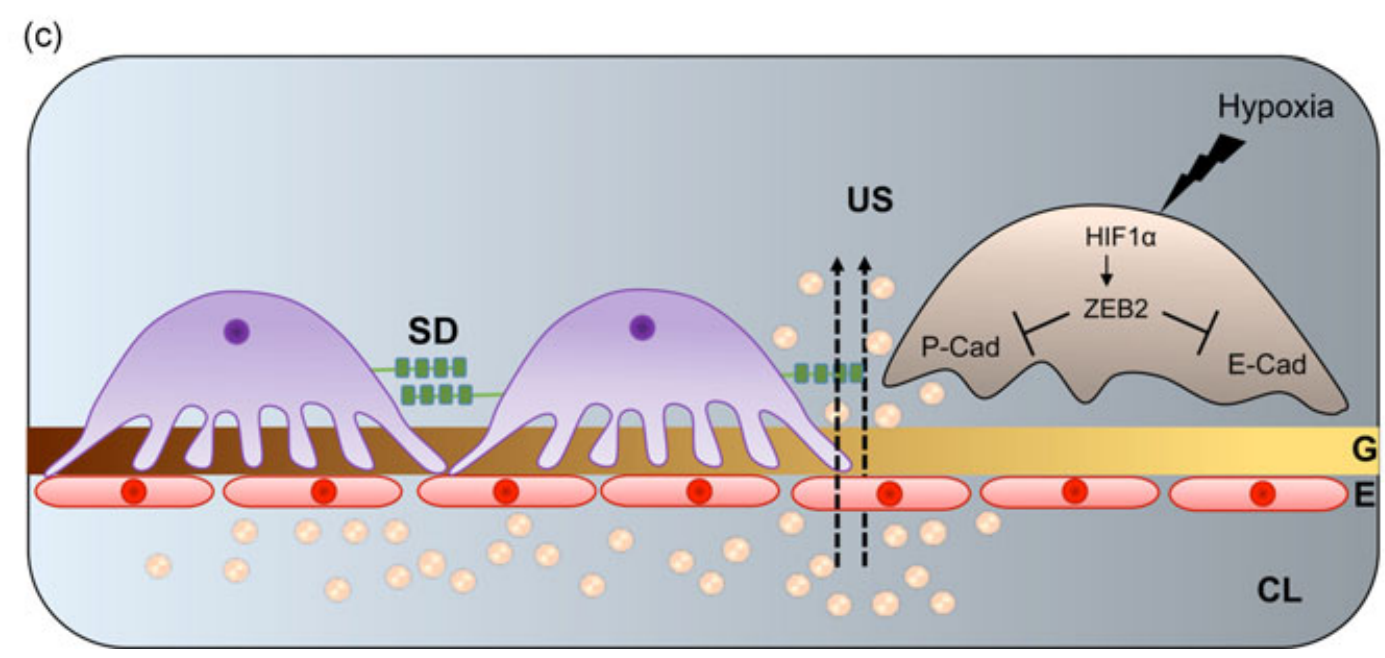

FIGURE 8 Activation of HIF1 $\alpha$-ZEB2 axis alters podocyte permselectivity. (a) A simplified schematic representation of the paracellular permeability assay adopted to assess the filtration barrier function of podocyte monolayer. Podocyte monolayer on collagen-coated transwell filters was allowed to differentiate as described in Section 2 and exposed to hypoxia for $24 \mathrm{hr}$. Albumin was placed in the bottom chamber, and its migration to the upper chamber was determined at respective intervals. (b) Quantification of albumin influx across podocyte monolayer. Albumin influx is determined at 1 and $4 \mathrm{hr}$ following the $24 \mathrm{hr}$ exposure to hypoxia. Podocytes transduced with a lentiviral construct expressing scrambled RNA were considered as a control. The data are presented as mean $\pm S E ; n=4-6 ;{ }^{*} p<0.05$. (c) Proposed model for hypoxia-mediated podocyte injury. Upon exposure to hypoxia, HIF1 $\alpha$ accumulates, which in turn occupies ZEB2 promoter and drives its expression. ZEB2, in turn, suppresses both P- and E-cadherin to induce EMT. Decreased E-cadherin expression is implicated in EMT that results in podocyte dehiscence and loss. These changes in P-and E-cadherin expression will manifest in effacement of podocyte foot-processes and proteinuria in pathological states of overactivity of HIF1 $\alpha$-ZEB2 axis in the kidney (CL, capillary lumen; US, urinary space; G-glomerular basement membrane; E-endothelium). EMT: epithelial-mesenchymal transition; HIF1 $\alpha$ : hypoxia-inducible factor-1 $\alpha$; shRNA: short hairpin RNA; ZEB2: zinc finger E-box binding homeobox 2 
HEK cells and human podocytes. In podocytes with a ZEB2 knockdown, HIF1 $\alpha$ accumulation fails to provoke the loss of E-cadherin; whereas ectopically expressed ZEB2 elicited further loss of E-cadherin expression. Furthermore, elevated ZEB2 expression altered the permselectivity of podocytes to albumin. These results indicate that ZEB2 is essential for transducing the HIF1 $\alpha$ effects on the permeability of the podocyte monolayer. In addition to ZEB2, there are other transcription factors that elicit EMT phenomenon including ZEB1, Snail, and Slug. We observed that basal level ZEB2 expression in podocytes was relatively much higher than compared with other transcription factors that induce EMT, while ZEB1 protein expression was only minimally increased during hypoxia (data not shown).

Besides the loss of E- and P-cadherin expression, several other proteins are also deregulated during EMT of podocytes including nephrin and podocin (Nishad et al., 2017). We found attenuated expression of ZO-1 and increased expression of $\mathrm{N}$-cadherin, $\beta$-catenin, and vimentin in rats exposed to hypoxia. ZO-1 is a component of tight junctions, which plays a vital role in regulating permeability across the epithelium. The decreased expression of ZO-1 in podocytes exposed to hypoxia could disrupt the permeability barrier of the podocytes. The loss of ZO-1 expression in our study is concurrent with earlier studies that reported the loss of tight junction proteins under ischemic conditions (Wagner et al., 2008). We have also observed the loss of podocyte-specific proteins nephrin and podocin in cells exposed to hypoxia. These two proteins are crucial components of podocyte slit-diaphragm, and loss of their expression, particularly nephrin, a major determinant of glomerular vascular permeability correlates with a loss of glomerular filter integrity (Hamano et al., 2002). Expression of nephrin and podocin is also decreased during EMT of podocytes (Li et al., 2008).

The results from this study show that ZEB2 expression is induced in podocytes under hypoxic conditions. We demonstrate that ZEB2 promoter has HREs and HIF1 $\alpha$ interacts with these HRE1 and HRE2 (5'-GCGTG-3' \& 5'-CACGT-3'). Activation of ZEB2 gene transcription is evidenced by an increase in ZEB2 promoter activity under hypoxic conditions. It is known that the expression of the ZEB2 is controlled at multiple levels, including transcriptional and posttranscriptional. Posttranscriptional regulation of ZEB2 is mediated by ZEB2-NAT, wherein ZEB2-NAT binds to a complementary region on ZEB2 mRNA. Binding of ZEB2-NAT with ZEB2 mRNA preserves internal ribosome entry site of ZEB2 mRNA by preventing splicing of an intron in ZEB2 mRNA. ZEB2-NAT mediated control of ZEB2 expression has been described in earlier studies (Beltran et al., 2008; Katoh \& Katoh, 2009). Interestingly, in addition to the ZEB2 expression, we identified the induction of ZEB2-NAT in hypoxic conditions. Our study reveals that a two-level regulation of ZEB2 expression by HIF1 $\alpha$ under hypoxic conditions. In addition to our own observations, meta-data analysis from Nephroseq also revealed that elevated expression of HIF1 $\alpha$ is associated with ZEB2 overexpression in CKD. Hence, increased HIF1 $\alpha$ and ZEB2 expression is not only confined to loss of glomerular function under hypoxic conditions but is also relevant to the pathogenesis of glomerular dysfunction in CKD.

This report establishes a mechanism for the effect of chronic hypoxia on glomerular dysfunction. A novel observation of this study is that hypoxia induces HIF1 $\alpha$, which in turn increases ZEB2 expression in the podocytes, and this action of HIF $1 \alpha$ is mediated, in part, via increased expression of ZEB2-NAT. The HIF1 $\alpha$ dependent increase in ZEB2 ensures both EMT of podocytes and podocyte foot-processes effacement. ZEB2 is critical for HIF1 $\alpha$ to exert its deleterious effect on glomerular function in hypoxia via changes in the epithelial phenotype and permselectivity of podocytes. We propose that the HIF1 $\alpha$-dependent increase in ZEB2 plays a key role in transducing the podocyte injury under hypoxic conditions and that the HIF1 $\alpha$-ZEB2 axis could be a molecular basis for podocyte loss and proteinuria during hypoxic conditions that prevail during several pathological and extreme physiological conditions.

\section{ACKNOWLEDGMENTS}

This study was funded by grants from Life Science Research Board (LSRB) and Science and Engineering Research Board (SERB) to A. K. P. A. K. P. is a recipient of DHR Fellowship from Government of India. Authors acknowledge UGC-SAP and UPE-2 sponsored infrastructural facility at Department of Biochemistry, University of Hyderabad. Research fellowship by ICMR (to K. N.) and UGC (to D. M.) are duly acknowledged.

\section{CONFLICTS OF INTEREST}

The authors declare that there are no conflicts of interest.

\section{ORCID}

Anil Kumar Pasupulati (D) http://orcid.org/0000-0001-9467-7650

\section{REFERENCES}

Adeseun, G. A., \& Rosas, S. E. (2010). The impact of obstructive sleep apnea on chronic kidney disease. Current Hypertension Reports, 12, 378-383.

Anil Kumar, P., Welsh, G. I., Saleem, M. A., \& Menon, R. K. (2014). Molecular and cellular events mediating glomerular podocyte dysfunction and depletion in diabetes mellitus. Frontiers in Endocrinology, 5, 151.

Babelova, A., Jansen, F., Sander, K., Löhn, M., Schäfer, L., Fork, C., ... Brandes, R. P. (2013). Activation of Rac-1 and RhoA contributes to podocyte injury in chronic kidney disease. PLOS One, 8, e80328.

Batlle, E., Sancho, E., Francí, C., Domínguez, D., Monfar, M., Baulida, J., \& García de herreros, A. (2000). The transcription factor snail is a repressor of E-cadherin gene expression in epithelial tumour cells. Nature Cell Biology, 2, 84-89.

Beltran, M., Puig, I., Pena, C., Garcia, J. M., Alvarez, A. B., Pena, R., ... de Herreros, A. G. (2008). A natural antisense transcript regulates Zeb2/ Sip1 gene expression during Snail1-induced epithelial-mesenchymal transition. Genes and Development, 22, 756-769. 
Boini, K. M., Xia, M., Xiong, J., Li, C., Payne, L. P., \& Li, P. L. (2012). Implication of CD38 gene in podocyte epithelial-to-mesenchymal transition and glomerular sclerosis. Journal of Cellular and Molecular Medicine, 16, 1674-1685.

Chadban, S. J., Briganti, E. M., Kerr, P. G., Dunstan, D. W., Welborn, T. A., Zimmet, P. Z., \& Atkins, R. C. (2003). Prevalence of kidney damage in Australian adults: The AusDiab kidney study. Journal of the American Society of Nephrology, 14, S131-S138.

Chitra, P. S., Swathi, T., Sahay, R., Reddy, G. B., Menon, R. K., \& Kumar, P. A. (2015). Growth hormone induces transforming growth factor-betainduced protein in podocytes: Implications for podocyte depletion and proteinuria. Journal of Cellular Biochemistry, 116, 1947-1956.

Christoffersen, N. R., Silahtaroglu, A., Orom, U. A., Kauppinen, S., \& Lund, A. H. (2007). MiR-200b mediates post-transcriptional repression of ZFHX1B. RNA, 13, 1172-1178.

Coresh, J., Astor, B. C., Greene, T., Eknoyan, G., \& Levey, A. S. (2003) Prevalence of chronic kidney disease and decreased kidney function in the adult US population: Third National Health and Nutrition Examination Survey. American Journal of Kidney Diseases, 41, 1-12.

Eckardt, K. U., Bernhardt, W. W., Weidemann, A., Warnecke, C. Rosenberger, C., Wiesener, M. M., \& Willam, C. (2005). Role of hypoxia in the pathogenesis of renal disease. Kidney International. Supplement, 68, S46-S51.

Fan, J., Fan, X., Li, Y., Ding, L., Zheng, Q., Guo, J., ... Gong, Y. (2016). Chronic normobaric hypoxia induces pulmonary hypertension in rats: Role of NF-kappaB. High Altitude Medicine \& Biology, 17, 43-49.

Fine, L. G., Bandyopadhay, D., \& Norman, J. T. (2000). Is there a common mechanism for the progression of different types of renal diseases other than proteinuria? Towards the unifying theme of chronic hypoxia. Kidney International. Supplement, 75, S22-S26.

Fine, L. G., \& Norman, J. T. (2008). Chronic hypoxia as a mechanism of progression of chronic kidney diseases: From hypothesis to novel therapeutics. Kidney International, 74, 867-872.

Forsythe, J. A., Jiang, B. H., Iyer, N. V., Agani, F., Leung, S. W., Koos, R. D., \& Semenza, G. L. (1996). Activation of vascular endothelial growth factor gene transcription by hypoxia-inducible factor 1. Molecular and Cellular Biology, 16, 4604-4613.

Franzén, S., Pihl, L., Khan, N., Gustafsson, H., \& Palm, F. (2016). Pronounced kidney hypoxia precedes albuminuria in type 1 diabetic mice. American Journal of Physiology. Renal Physiology, 310, F807-F809.

Fu, Q., Colgan, S. P., \& Shelley, C. S. (2016). Hypoxia: The force that drives chronic kidney disease. Clinical Medicine \& Research, 14, 15-39.

Goldfarb-Rumyantzev, A. S., \& Alper, S. L. (2014). Short-term responses of the kidney to high altitude in mountain climbers. Nephrology, Dialysis, Transplantation, 29, 497-506.

van Grunsven, L. A., Michiels, C., Van de Putte, T., Nelles, L., Wuytens, G., Verschueren, K., \& Huylebroeck, D. (2003). Interaction between Smad-interacting protein-1 and the corepressor C-terminal binding protein is dispensable for transcriptional repression of $\mathrm{E}$-cadherin. The Journal of Biological Chemistry, 278, 26135-26145.

Haase, V. H. (2013). Mechanisms of hypoxia responses in renal tissue. Journal of the American Society of Nephrology, 24, 537-541.

Hajra, K. M., Chen, D. Y., \& Fearon, E. R. (2002). The SLUG zinc-finger protein represses E-cadherin in breast cancer. Cancer Research, 62, 1613-1618.

Hamano, Y., Grunkemeyer, J. A., Sudhakar, A., Zeisberg, M., Cosgrove, D., Morello, R., ... Kalluri, R. (2002). Determinants of vascular permeability in the kidney glomerulus. The Journal of Biological Chemistry, 277, 31154-31162.

Hansell, P., Welch, W. J., Blantz, R. C., \& Palm, F. (2013). Determinants of kidney oxygen consumption and their relationship to tissue oxygen tension in diabetes and hypertension. Clinical and Experimental Pharmacology \& Physiology, 40, 123-137.

Hartner, A., Porst, M., Gauer, S., Pröls, F., Veelken, R., \& Hilgers, K. F. (2001). Glomerular osteopontin expression and macrophage infiltration in glomerulosclerosis of DOCA-salt rats. American Journal of Kidney Diseases, 38, 153-164.

Katoh, M., \& Katoh, M. (2009). Integrative genomic analyses of ZEB2: Transcriptional regulation of ZEB2 based on SMADs, ETS1, HIF1alpha, POU/OCT, and NF-kappaB. International Journal of Oncology, 34, 1737-1742.

Krtil, J., Pláteník, J., Kazderová, M., Tesar, V., \& Zima, T. (2007). Culture methods of glomerular podocytes. Kidney \& Blood Pressure Research, 30, 162-174.

Kumar, P. A., Kotlyarevska, K., Dejkhmaron, P., Reddy, G. R., Lu, C., Bhojani, M. S., \& Menon, R. K. (2010). Growth hormone (GH)dependent expression of a natural antisense transcript induces zinc finger E-box-binding homeobox 2 (ZEB2) in the glomerular podocyte: A novel action of gh with implications for the pathogenesis of diabetic nephropathy. The Journal of Biological Chemistry, 285, 31148-31156.

Kushida, N., Nomura, S., Mimura, I., Fujita, T., Yamamoto, S., Nangaku, M., \& Aburatani, H. (2016). Hypoxia-inducible factor-1alpha activates the transforming growth factor-beta/SMAD3 pathway in kidney tubular epithelial cells. American Journal of Nephrology, 44, 276-285.

Li, Y., Kang, Y. S., Dai, C., Kiss, L. P., Wen, X., \& Liu, Y. (2008). Epithelial-tomesenchymal transition is a potential pathway leading to podocyte dysfunction and proteinuria. American Journal of Pathology, 172, 299-308.

Majmundar, A. J., Wong, W. J., \& Simon, M. C. (2010). Hypoxia-inducible factors and the response to hypoxic stress. Molecular Cell, 40, 294-309.

Mungamuri, S. K., Wang, S., Manfredi, J. J., Gu, W., \& Aaronson, S. A. (2015). Ash2L enables P53-dependent apoptosis by favoring stable transcription pre-initiation complex formation on its pro-apoptotic target promoters. Oncogene, 34, 2461-2470.

Munshi, R., Hsu, C., \& Himmelfarb, J. (2011). Advances in understanding ischemic acute kidney injury. BMC Medicine, 9, 11.

Nangaku, M. (2011). Hypoxia in chronic kidney disease: the final common pathway to end stage renal disease. In T. Miyata, K. -U. Eckardt, \& M. Nangaku (Eds.), Studies on Renal Disorders (pp. 545-557). Totowa, NJ: Humana Press.

Ni, L., Saleem, M., \& Mathieson, P. W. (2012). Podocyte culture: Tricks of the trade. Nephrology, 17, 525-531.

Nishad, R. , K., Nakuluri, M., Motrapu, A. K., \& Pasupulati, A. K. (2017). Epithelial-mesenchymal transition of glomerular podocytes: Implications in proteinuria. MGM Journal of Medical Science, 4(1), 1-9.

O'Brien, S. P., Smith, M., Ling, H., Phillips, L., Weber, W., Lydon, J., ... Wawersik, S. (2013). Glomerulopathy in the KK.Cg-A(y) /J mouse reflects the pathology of diabetic nephropathy. Journal of Diabetes Research, 2013, 498925-13.

Palacios, F., Tushir, J. S., Fujita, Y., \& D'Souza-Schorey, C. (2005). Lysosomal targeting of E-cadherin: A unique mechanism for the down-regulation of cell-cell adhesion during epithelial to mesenchymal transitions. Molecular and Cellular Biology, 25, 389-402.

Palm, F., \& Nordquist, L. (2011). Renal tubulointerstitial hypoxia: Cause and consequence of kidney dysfunction. Clinical and Experimental Pharmacology \& Physiology, 38, 474-480.

Rasouly, H. M., Kumar, S., Chan, S., Pisarek-Horowitz, A., Sharma, R., Xi, Q. J., ... Lu, W. (2016). Loss of Zeb2 in mesenchyme-derived nephrons causes primary glomerulocystic disease. Kidney International, 90, 1262-1273.

Reiser, J., Kriz, W., Kretzler, M., \& Mundel, P. (2000). The glomerular slit diaphragm is a modified adherens junction. Journal of the American Society of Nephrology, 11, 1-8.

Stojanović, V. R., Jovanović, I. D., Ugrenović, S. Z., Vasović, L. P., Živković, V. S., Jocić, M. V., ... Pavlović, M. N. (2012). Morphometric analysis of nonsclerosed Glomeruli size and connective tissue content during the aging process. The Scientific World Journal, 2012, 845046-845048.

Tanaka, S., Tanaka, T., \& Nangaku, M. (2016). Hypoxia and hypoxiainducible factors in chronic kidney disease. Renal Replacement Therapy, 2, 25. 
Tanaka, T., Miyata, T., Inagi, R., Fujita, T., \& Nangaku, M. (2004). Hypoxia in renal disease with proteinuria and/or glomerular hypertension. The American Journal of Pathology, 165, 1979-1992.

Tao, Y., Dong, W., Li, Z., Chen, Y., Liang, H., Li, R., ... Liang, X. (2016). Proteinuria as an independent risk factor for contrast-induced acute kidney injury and mortality in patients with stroke undergoing cerebral angiography. Journal of Neurointerventional Surgery, 9, 445-448.

Vandewalle, C., Comijn, J., De Craene, B., Vermassen, P., Bruyneel, E., Andersen, H., ... Berx, G. (2005). SIP1/ZEB2 induces EMT by repressing genes of different epithelial cell-cell junctions. Nucleic Acids Research, 33, 6566-6578.

Wagner, M. C., Rhodes, G., Wang, E., Pruthi, V., Arif, E., Saleem, M. A., ... Nihalani, D. (2008). Ischemic injury to kidney induces glomerular podocyte effacement and dissociation of slit diaphragm proteins Neph1 and ZO-1. The Journal of Biological Chemistry, 283, 35579-35589.

Wang, G. L., \& Semenza, G. L. (1993). Characterization of hypoxiainducible factor 1 and regulation of DNA binding activity by hypoxia. The Journal of Biological Chemistry, 268, 21513-21518.
Wheelock, M. J., Shintani, Y., Maeda, M., Fukumoto, Y., \& Johnson, K. R. (2008). Cadherin switching. Journal of Cell Science, 121, 727-735.

Wight, M., \& Werner, A. (2013). The functions of natural antisense transcripts. Essays in Biochemistry, 54, 91-101.

Wiles, E. T., Bell, R., Thomas, D., Beckerle, M., \& Lessnick, S. L. (2013). ZEB2 Represses the epithelial phenotype and facilitates metastasis in Ewing sarcoma. Genes \& Cancer, 4, 486-500.

Xu, Z. G., Ryu, D. R., Yoo, T. H., Jung, D. S., Kim, J. J., Kim, H. J., ... Kang, S. W. (2005). P-Cadherin is decreased in diabetic glomeruli and in glucose-stimulated podocytes in vivo and in vitro studies. Nephrology, Dialysis, Transplantation, 20, 524-531.

How to cite this article: Nakuluri K, Mukhi D, Nishad R, et al. Hypoxia induces ZEB2 in podocytes: Implications in the pathogenesis of proteinuria. J Cell Physiol. 2019;234:

6503-6518. https://doi.org/10.1002/jcp.27387 